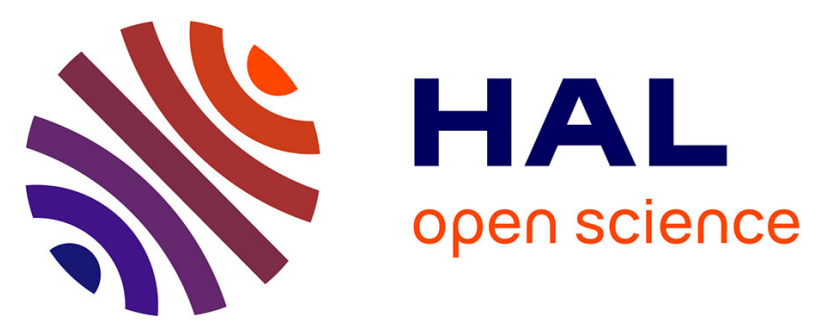

\title{
Evaluation du potentiel d'émission d'alkylphénols et de bisphénol A par lessivage des matériaux de construction, des pièces et des consommables automobiles
}

Katerine Lamprea, Cécile Mirande-Bret, Adèle Bressy, Marie-Christine Gromaire

\section{To cite this version:}

Katerine Lamprea, Cécile Mirande-Bret, Adèle Bressy, Marie-Christine Gromaire. Evaluation du potentiel d'émission d'alkylphénols et de bisphénol A par lessivage des matériaux de construction, des pièces et des consommables automobiles. Techniques Sciences Méthodes , 2017, 7/8 (7-8), pp.71-90. 10.1051/tsm/201778071. hal-01621269

HAL Id: hal-01621269

https://hal-enpc.archives-ouvertes.fr/hal-01621269

Submitted on 23 May 2018

HAL is a multi-disciplinary open access archive for the deposit and dissemination of scientific research documents, whether they are published or not. The documents may come from teaching and research institutions in France or abroad, or from public or private research centers.
L'archive ouverte pluridisciplinaire HAL, est destinée au dépôt et à la diffusion de documents scientifiques de niveau recherche, publiés ou non, émanant des établissements d'enseignement et de recherche français ou étrangers, des laboratoires publics ou privés. 


\begin{tabular}{|c|c|}
\hline $\begin{array}{l}\text { Lamprea } \mathrm{K}^{1}{ }^{1}, \text { Mirande-Bret } \mathrm{C}^{1}{ }^{1} \text {, } \\
\text { Bressy A. }{ }^{1}, \text { Caupos E. }{ }^{1}, \\
\text { Gromaire M.C. }{ }^{1{ }^{*}}\end{array}$ & $\begin{array}{c}\text { Évaluation du potentiel d'émission } \\
\text { d'alkylphénols et de bisphénol A par } \\
\text { lessivage des matériaux de construction, } \\
\text { des pièces et des consommables } \\
\text { automobiles. }\end{array}$ \\
\hline $\begin{array}{l}{ }^{1} \text { LEESU, UMR-MA } 102 \text { - École des } \\
\text { Ponts ParisTech, UPEC, } \\
\text { AgroParisTech, 6-8 avenue Blaise } \\
\text { Pascal Cité Descartes, } 77455 \\
\text { Champs-sur-Marne, France : }{ }^{*} \text { marie- } \\
\text { christine.gromaire@enpc.fr }\end{array}$ & $\begin{array}{l}\text { TSM, 7-8 (2017) 71-90 } \\
\text { DOI: https://doi.org/10.1051/tsm/201778071 }\end{array}$ \\
\hline
\end{tabular}

\section{RESUME}

La contamination en alkylphénols (AP) et en bisphénol A (BPA) des eaux de ruissellement urbaine est avérée. Les sources potentielles de ces contaminants dans les eaux de ruissellement sont endogènes au bassin versant et sont liées en particulier à la circulation automobile et au lessivage des matériaux constituant l'enveloppe bâtie et les infrastructures urbaines. Cet article synthétise les résultats d'un travail expérimental mené sur une sélection de matériaux de construction, de matériaux et de consommables automobiles entrant en contact avec la pluie afin d'évaluer leur potentiel d'émission d'alkylphénols, alkylphénols éthoxylés et bisphénol A dans le ruissellement. 36 échantillons de matériaux, neufs et usagés, appartenant à 7 grandes familles de matériaux de construction (PVC, béton, polycarbonate, bitume modifié SBS, matériaux de drainage) et de matériaux automobiles (carrosserie, pneus) ont été soumis à des tests de lixiviation au méthanol puis, pour une sélection d'entre eux, à des tests de lixiviation à l'eau. 3 types de fluides automobiles (liquide de frein, lave-glace et liquide de refroidissement) ont été extraits directement. Les résultats démontrent la présence ubiquiste des composés recherchés dans les matériaux urbains, ainsi que leur caractère extractible à l'eau. Les composés avec les plus forts taux d'émission sont le bisphénol $A$ et le nonylphénol. Les émissions dans l'eau en bisphénol $A$ les plus importantes (10 à $300 \mathrm{ng} / \mathrm{g}$ ) ont été mesurées pour le polycarbonate, les pneumatiques, certaines carrosseries de voiture et les PVC. Le nonylphénol a été lixivié par l'eau en quantité importante (1 à $10 \mathrm{ng} / \mathrm{g}$ ) depuis les PVC, certains bétons, les bitumes modifiés SBS et les échantillons de carrosserie. Les pneus sont les seuls matériaux présentant une forte émission en octylphénol (1 à $10 \mathrm{ng} / \mathrm{g}$ ). L'analyse de fluides automobiles a confirmé la présence de BPA $(0,3$ à $5,5 \mathrm{~g} / \mathrm{L})$ et de nonylphénol $(2,3$ à $2,9 \mathrm{mg} / \mathrm{L})$ dans les liquides de frein, tandis qu'AP et BPA peuvent être trouvés à l'état de traces dans les liquides de refroidissement et lave-glaces.

\section{MOTS-CLES}

Alkylphénols, Bisphénol A, Émissions, Lixiviation, Matériaux du bâtiment, Matériaux de l'automobile, Ruissellement 


\section{INTRODUCTION}

Les alkylphénols (AP) et le bisphénol $A(B P A)$ sont des molécules de synthèse qui ne sont pas présentes à l'état naturel. Leur présence dans l'air, les sols et l'eau est donc le résultat d'émissions générées par des activités anthropiques. Les études épidémiologiques conduites sur ces molécules mettent en évidence leur potentiel toxicologique en tant que perturbateurs endocriniens chez l'Homme et chez l'animal (Harréus et al. 2002; Soares et al. 2008; Benachour and Aris 2009; ANSES 2011).

Depuis quelques années la réduction de l'exposition de l'Homme aux substances cataloguées en tant que perturbateurs endocriniens est devenue un sujet d'intérêt au sein de l'Union Européenne. De ce fait, la mise en place de nouvelles réglementations et la réalisation d'accords volontaires avec les industriels sont en train de conduire à une diminution progressive voire à la restriction d'utilisation de certaines de ces substances. C'est le cas du nonylphénol (NP) et des nonylphénols éthoxylés (NPEO), dont l'utilisation a été interdite par la Directive Européenne 2003/53/CE (Union européenne 2003) à une concentration supérieure à 0,1\% pour différents usages (produits de nettoyage, traitement des textiles...). En ce qui concerne le BPA, le règlement européen 321/2011 (2011) a interdit la fabrication de biberons en polycarbonate (matériau fabriqué à base de BPA) depuis le premier avril 2011 ainsi que leur importation et mise sur le marché depuis le premier juin 2011. Depuis le 1 janvier 2015, la loi française $n^{\circ}$ 2012-1442 (2012) suspend la fabrication, l'importation, l'exportation et la mise sur le marché à titre gratuit ou onéreux de tout conditionnement, contenant ou ustensile comportant du bisphénol $A$ et destiné à entrer en contact direct avec toutes les denrées alimentaires. Enfin, le nonylphénol (NP) et l'octylphénol (OP) sont inscrits sur la liste des substances prioritaires de la Directive cadre sur l'Eau 2000/60/CE (Union européenne 2000). Malgré ces efforts, les secteurs d'application pour les AP et le BPA restent relativement nombreux, et leurs émissions dans l'environnement sont donc loin d'être supprimées.

La présence des AP et du BPA dans les eaux de ruissellement en milieu urbain a été reportée dans plusieurs études, à des concentrations cependant variables (Fries and Püttmann 2004; Rule et al. 2006; Eriksson et al. 2007; Björklund et al. 2009; Becouze-Lareure 2010; Bressy et al. 2011; Bressy et al. 2012). Les analyses réalisées dans le cadre du projet INOGEV sur trois bassins versants urbains d'occupations du sol variées (Gasperi ce numéro; Gasperi et al. 2014) ont démontré la présence ubiquiste des alkylphénols et du bisphénol A dans les eaux pluviales urbaines et leur origine endogène aux bassins versants étudiés. Pour les NP et l'OP, les concentrations observées sont généralement supérieures aux Normes de Qualité Environnementales (NQE) fixées respectivement à 0,3 et $0,1 \mu \mathrm{g} \cdot \mathrm{L}^{-1}$ (Directive Européenne 2003/53/CE).

Les auteurs s'accordent à dire qu'en milieu urbain les matériaux de construction (béton, peintures, plastiques) ainsi que le secteur automobile sont des voies importantes d'introduction des AP dans les eaux pluviales (Björklund et al. 2009; Bressy et al. 2011; Gasperi et al. 2014). Cependant, compte tenu de la diversité de formulation des matériaux en fonction de leur usage et du caractère très général des données de la littérature, il est très difficile d'identifier avec précision les sources primaires contaminant les eaux de ruissellement et de les hiérarchiser en fonction de leur contribution relative. De telles informations seraient pourtant nécessaires afin d'orienter des actions préventives ou incitatives permettant de réduire les émissions à la source, mais également pour alimenter les modèles d'analyse de flux de substances (Substances Flow Analysis en anglais ou SFA) à l'échelle urbaine.

L'objectif de ce travail est de réaliser une évaluation expérimentale du potentiel d'émission dans l'eau des AP et BPA pour une sélection de matériaux urbains, de pièces et de fluides automobiles pour lesquels la présence de ces composés est suspectée. II s'est inscrit à la fois dans le projet INOGEV, financé par l'ANR, portant sur la gestion durable des eaux urbaines, et le projet RGCU TVGEP portant sur l'utilisation des toitures végétalisées comme outil de gestion des eaux pluviales. Les matériaux et produits testés ont donc été sélectionnés du fait de leur prévalence dans l'environnement urbain, avec un focus sur les matériaux utilisés pour la structure des couvertures végétalisées. 29 matériaux de construction neufs et usagés, 7 pièces automobiles et 11 échantillons de fluides automobiles ont été étudiés. Sur ces matériaux, des essais de lixiviation au méthanol ont été réalisés pour identifier la présence ou non d'AP et BPA extractibles, puis pour une sélection d'entre eux des essais de lixiviation à l'eau ont été conduits afin d'établir un profil d'émission des différents matériaux en fonction des niveaux de concentrations mesurés dans le lixiviat d'eau et des masses extraites par unité de masse de matériau. 


\section{METHODOLOGIE}

\subsection{Matériaux testés}

\section{Critères de sélection}

La sélection de matériaux a été effectuée en prenant en compte :

- les données issues de la littérature sur l'utilisation des AP et du BPA ;

- les données de littérature grise sur la composition de l'enveloppe automobile et des fluides ;

- des données sur les matériaux les plus employés dans le marché français de couvertures de toit, des menuiseries et de façades.

Selon la littérature, les secteurs d'utilisation des AP et BPA dont les produits fabriqués sont susceptibles d'être en contact direct avec de l'eau pluie, sont très diversifiés. Le Tableau 1 récapitule ces différentes applications.

Tableau 1 : Utilisations des AP et BPA susceptibles de rentrer en contact avec de l'eau de pluie (Hellgren et al. 1999; ECB 2002; OSPAR Commission 2003; ECB 2010; INERIS 2010; ANSES 2011; INERIS 2011; INERIS 2016)

\begin{tabular}{|c|c|}
\hline NP : secteurs d'activité & Applications \\
\hline $\begin{array}{l}\text { Fabrication de NPEO } \\
\text { (nonylphénols éthoxylés) }\end{array}$ & 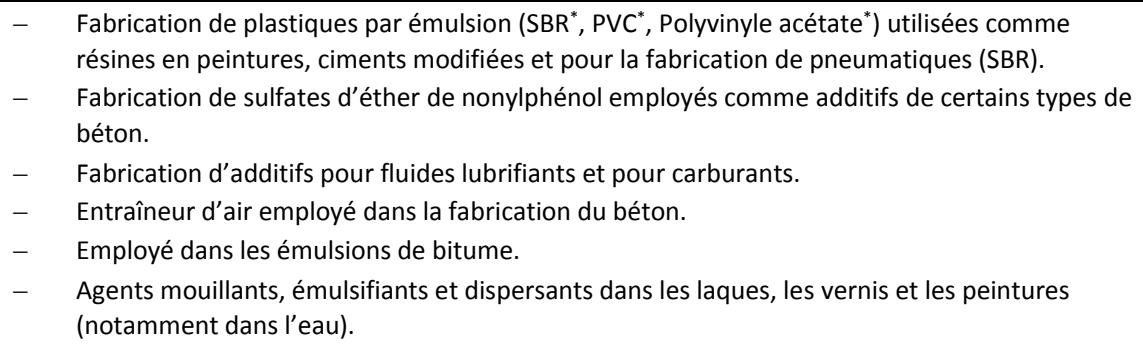 \\
\hline $\begin{array}{l}\text { Production de résines } \\
\text { phénoliques }\end{array}$ & - $\quad$ Agent d'adhérence dans le caoutchouc des pneus (SBR, EPDM*, BR*). \\
\hline $\begin{array}{l}\text { Production de résines époxydes } \\
\text { (Catalyseur dans le } \\
\text { durcissement) }\end{array}$ & $\begin{array}{l}\text { - Employés comme revêtement de sols, sols composites, armatures en acier des bâtiments (ex: } \\
\text { panneau sandwich) et de surfaces métalliques en contact avec l'eau. } \\
\text { - Stabilisant de peintures à base de résines vinyliques utilisées pour les façades, toitures et } \\
\text { comme peintures anti-corrosion. }\end{array}$ \\
\hline $\begin{array}{l}\text { Production de TNPP } \\
\text { (trinonylphénol phosphite). }\end{array}$ & $\begin{array}{l}\text { - Stabilisateur pour caoutchoucs naturels et synthétiques (SBR, EPDM), polymères vinyliques, } \\
\text { polystyrène, polycarbonate. }\end{array}$ \\
\hline OP : secteurs d'activité & Applications \\
\hline $\begin{array}{l}\text { Fabrication des OPEO } \\
\text { (octylphénols éthoxylés) }\end{array}$ & $\begin{array}{l}\text { - Agent émulsifiant pour la synthèse de polymères comme les acryliques et le vinylacrylique } \\
\text { employés comme résines pour peintures de façades, toitures, anti-corrosion. }\end{array}$ \\
\hline $\begin{array}{l}\text { Production de résines } \\
\text { formophénoliques }\end{array}$ & - $\quad$ Agent d'adhérence dans le caoutchouc des pneus. \\
\hline BPA : secteurs d'activité & Applications \\
\hline Production de polycarbonate & $\begin{array}{l}\text { - Secteur de la construction : fabrication de couverture de toits, vérandas, bardages, panneaux } \\
\text { solaires, serres, } \\
\text { - } \quad \text { Secteur automobile : les phares avant de voitures, les pare-chocs, les pare-brises de motos, les } \\
\text { modules de toit des voitures convertibles, grilles de radiateur. }\end{array}$ \\
\hline Production de résines époxy & $\begin{array}{l}\text { - Employés comme revêtement de sols, sols composites, armatures en acier des bâtiments et } \\
\text { de surfaces métalliques. } \\
\text { - } \quad \text { Stabilisant de peintures à base de résines vinyliques utilisées pour les façades, toitures et } \\
\text { comme peintures anti-corrosion dans les véhicules (sous-couche). }\end{array}$ \\
\hline $\begin{array}{l}\text { Antioxydant et inhibiteur dans } \\
\text { la production de PVC* }\end{array}$ & $\begin{array}{l}\text { - Employé dans la fabrication de plusieurs matériaux de construction tels que fenêtres, portes, } \\
\text { revêtements, tuyaux, gouttières, volets. }\end{array}$ \\
\hline $\begin{array}{l}\text { Production de TBBPA } \\
\text { (Tétrabromobisphénol A) }\end{array}$ & - $\quad$ Retardateur de flamme et antioxydant dans les liquides de frein. \\
\hline $\begin{array}{l}\text { Production de résines } \\
\text { phénoliques }\end{array}$ & - $\quad$ Agent d'adhérence dans le caoutchouc des pneus (SBR, EPDM, BR). \\
\hline Industrie de peintures & Durcisseur de peintures. \\
\hline Industrie automobile & $\begin{array}{ll}- & \text { Ajouté à des liquides de frein à une concentration inférieure à } 1 \% . \\
- & \text { Antioxydant dans la matrice de polymère de pneus. } \\
\text { - } & \text { Ajouté aux produits d'entretiens de véhicules à une concentration de 0,3 - } 1 \% .\end{array}$ \\
\hline
\end{tabular}

*SBR : caoutchouc styrène-butadiène, EPDM : Ethylène propylène diène monomère, BR : caoutchouc polybutadiène, PVC : chlorure de polyvinyle 
En ce qui concerne les matériaux de construction (couverture des toits, fenêtres et façades) dans le marché français, les études de marché consultées (MSI Reports Ltd 2006; MSI Reports Ltd 2007; MSI Reports Ltd 2009; MSI Reports Ltd 2010; MSI Reports Ltd 2011) mettent en évidence que le PVC, l'aluminium, l'acier, le polycarbonate ainsi que les membranes d'étanchéité SBS sont des matériaux largement employés. Le PVC représente ainsi $62 \%$ du marché des fenêtres, $54 \%$ des volets et $21 \%$ des portes (MSI Reports Ltd 2011). Le polycarbonate est utilisé dans $27 \%$ des toitures de vérandas. Les membranes bitumineuses représentaient $74 \%$ du marché des produits d'étanchéité-toiture en 2005, dont $80 \%$ de membranes bitumineuses SBS et $20 \%$ de membranes bitumineuses APP. L'aluminium et l'acier sont largement présents dans le secteur résidentiel, commercial et industriel au niveau des couvertures, bardages, menuiseries. Il s'agit généralement de métaux prélaqués, dont la surface est couverte d'un revêtement organique.

\section{Description des matériaux testés}

Au total, 36 échantillons correspondant à 7 grandes familles de matériaux utilisés ont été testés dans cette étude (Tableau 2). II s'agit du PVC (8 échantillons), du béton (12 échantillons), des membranes d'étanchéité SBS (4 échantillons), de matériaux de drainage et filtration employés dans les toitures végétalisées (4 échantillons), du polycarbonate (1 échantillon), de l'acier laqué (4 échantillons), de pneumatiques (3 échantillons). Les échantillons testés comprenaient aussi bien des matériaux neufs qu'usagés. Il s'agissait pour les produits usagés, d'évaluer l'impact du stock des matériaux en place dans l'enveloppe bâtie, et pour les matériaux neufs, d'évaluer l'impact de l'évolution des réglementations concernant l'utilisation des substances étudiées.

Matériaux en PVC. Cinq descentes de gouttières (trois neuves, deux usagées), une canalisation usagée et deux volets roulants (un neuf et un usagé) ont été sélectionnés. Dans le domaine de l'évacuation des eaux pluviales, le PVC utilisé pour la fabrication des tuyaux et raccords est généralement du PVC non plastifié ou PVC-U. Les gouttières en PVC sont de couleur gris pastel ou beige.

Bétons. Dix blocs de béton, une dalle et une tuile en béton de provenances diverses ont été sélectionnés. Parmi les blocs en béton, quatre correspondent à des blocs provenant de trottoirs démolis, quatre autres sont des blocs issus de bâtiments démolis. Les deux restants proviennent respectivement d'un bâtiment en construction (neuf) et d'un réseau d'assainissement (ancien). La dalle de béton est un matériau neuf. La tuile en béton choisie est une tuile usagée d'environ une quinzaine d'années dont la surface présente une couche de revêtement écaillée. Membrane d'étanchéité SBS. Ces matériaux sont constituées d'une armature en fibres de polyester non-tissé et de bitume élastomère composé par un mélange de bitume sélectionné et de polymères thermoplastiques SBS. La masse bitumineuse peut contenir des agents anti-racines empêchant la pénétration des racines à travers le complexe étanche. La face supérieure est protégée par une autoprotection minérale " paillettes ardoise " et la face inférieure est recouverte d'un film thermo-fusible.

Pneumatiques. Trois pneumatiques de voiture et un pneumatique de moto usagés ont été collectés. Les pneumatiques testés sont de marques différentes.

Panneau en polycarbonate. Le panneau en polycarbonate est un panneau alvéolaire neuf à multiples parois d'épaisseur $16 \mathrm{~mm}$ et de masse approximative $2,7 \mathrm{~kg} / \mathrm{m}^{2}$. II est revêtu d'un film d'absorption des rayons UV.

Matériaux de drainage et filtration. Trois types de matériaux de drainage neufs et un matériau filtrant neuf utilisés en toiture végétalisée ont été testés. Le premier est un drainage en nid d'abeille de maille $50 \mathrm{~mm}$ en polypropylène noir issu à $60 \%$ de polypropylène recyclé. II présente sur une face un parement synthétique (polyester noir) résistant et filtrant. Le second est un drainage en polystyrène expansé alvéolé et perforé de 36 $\mathrm{mm}$ d'épaisseur avec une densité de $25 \mathrm{~kg} / \mathrm{m}^{3}$ et une masse de $0,7 \mathrm{~kg} / \mathrm{m}^{2}$. Le troisième matériau est une natte de drainage en PO/PE composée d'un tissu géotextile monofilament léger en polyamide (PO) doublé et d'une natte filtrante non tissée en polyamide et polyéthylène (PE). Elle a une épaisseur de $20 \mathrm{~mm}$ et une masse surfacique totale de $0,5 \mathrm{~kg} / \mathrm{m}^{2}$. La couche filtrante testée est un géotextile non-tissé de fibres synthétiques continues en polypropylène. Les fibres sont uniformément réparties et thermosoudées. La masse surfacique de ce matériau est de $0,1 \mathrm{~kg} / \mathrm{m}^{2}$.

Acier peint/laqué. Les échantillons étudiés correspondent pour trois d'entre eux à de la carrosserie de voiture et pour le quatrième à une tôle d'acier galvanisé laqué neuve (simple peau et avec nervures saillantes, épaisseur $0,75 \mathrm{~mm}$, masse $6 \mathrm{~kg} / \mathrm{m}^{2}$ ) utilisée en construction. Les morceaux de carrosseries ont été prélevés sur des véhicules différents et à des endroits différents de la carrosserie : portière d’un véhicule de 1989 peint en couleur 
bleue métallisée, toit d’un véhicule de 2001 peint de couleur bleue métallisée, capot de couleur blanc non métallisée, année du véhicule inconnue.

Par ailleurs, trois types de fluides automobiles ont été analysés : liquide de frein (3 échantillons), lave-glace (4 échantillons) et liquide de refroidissement (4 échantillons). Ces fluides ont été achetés en grande surface, en sélectionnant une diversité de marques, allant du premier prix au haut de gamme. Les huiles moteur et carburants, bien que sources potentielles d'AP (Diehl et al. 2004), n'ont pas été étudiées dans ce travail du fait de difficultés analytiques, notre protocole n'étant pas adapté à ce type de matrices.

\section{Récapitulatif des tests réalisés}

Tous les matériaux ont été soumis à des essais de lixiviation au méthanol en conditions statiques afin de déterminer la présence ou l'absence des AP et du BPA extractible. Par la suite, une sélection de matériaux, pour lesquels la présence des AP et/ou du BPA a été prouvée par les essais de lixiviation au méthanol, a été soumise à des essais de lixiviation dans l'eau. Ces essais ont eu comme objectif d'établir un profil d'émission des matériaux dans l'eau.

Les fluides automobiles ont quant à eux été extraits directement, afin de déterminer leurs teneurs en AP et BPA.

Tableau 2 : Liste récapitulative des matériaux testés en lixiviation au méthanol et à l'eau

\begin{tabular}{|c|c|c|c|c|c|c|c|}
\hline $\mathbf{N}^{\circ}$ & Type de matériau & $\begin{array}{c}\text { Lixiviation au } \\
\text { méthanol }\end{array}$ & $\begin{array}{l}\text { Lixiviation à } \\
\text { l'eau }\end{array}$ & $\mathbf{N}^{\circ}$ & Type de matériau & $\begin{array}{c}\text { Lixiviation au } \\
\text { méthanol }\end{array}$ & $\begin{array}{l}\text { Lixiviation à } \\
\text { l'eau }\end{array}$ \\
\hline 1 & Gouttière en PVC neuve 1 & $x$ & $x$ & 19 & Dalle de béton & $x$ & \\
\hline 2 & Gouttière en PVC neuve 2 & $x$ & $x$ & 20 & Tuile en béton usagée & $x$ & \\
\hline 3 & Gouttière en PVC neuve 3 & $\mathrm{x}$ & $\mathrm{x}$ & 21 & $\begin{array}{l}\text { Panneau en } \\
\text { polycarbonate }\end{array}$ & & $x$ \\
\hline 4 & Gouttière en PVC usagé & $\mathrm{x}$ & $\mathrm{x}$ & 22 & $\begin{array}{l}\text { Membrane d'étanchéité } \\
\text { SBS } 1\end{array}$ & $\mathrm{x}$ & $\mathrm{x}$ \\
\hline 5 & $\begin{array}{l}\text { Gouttière en PVC usagé } \\
\text { (surface peinte) }\end{array}$ & $x$ & $x$ & 23 & $\begin{array}{l}\text { Membrane d'étanchéité } \\
\text { SBS } 2\end{array}$ & $x$ & \\
\hline 6 & Canalisation PVC usagé & $x$ & & 24 & $\begin{array}{l}\text { Membrane d'étanchéité } \\
\text { SBS } 3\end{array}$ & $x$ & $x$ \\
\hline 7 & Volet en PVC neuf & $x$ & $x$ & 25 & Pneumatique moto & $x$ & \\
\hline 8 & Volet en PVC usagé & $x$ & $x$ & 26 & Pneumatique voiture 1 & $x$ & $x$ \\
\hline 9 & Béton de trottoir usagé 1 & $x$ & & 27 & Pneumatique voiture 2 & $x$ & $x$ \\
\hline 10 & Béton de trottoir usagé 2 & $x$ & $x$ & 28 & Pneumatique voiture 3 & $x$ & \\
\hline 11 & Béton de trottoir usagé 3 & $x$ & & 29 & Plaque en acier laqué & $x$ & $x$ \\
\hline 12 & Béton de trottoir usagé 4 & $x$ & $x$ & 30 & Carrosserie 1 & $x$ & $x$ \\
\hline 13 & Béton construction usagé 1 & $x$ & & 31 & Carrosserie 2 & $x$ & $x$ \\
\hline 14 & Béton de construction usagé 2 & $x$ & & 32 & Carrosserie 3 & $x$ & $x$ \\
\hline 15 & Béton de construction usagé 3 & $x$ & & 33 & $\begin{array}{l}\text { Couche de drainage } \\
\text { polypropylène }\end{array}$ & $x$ & $x$ \\
\hline 16 & Béton de construction neuf & $x$ & & 34 & $\begin{array}{l}\text { Natte de drainage } \\
\text { polyamide/ polyéthylène } \\
\text { (PO/PE) }\end{array}$ & $x$ & \\
\hline 17 & Béton de réseau & $x$ & & 35 & $\begin{array}{l}\text { Couche drainage } \\
\text { polystyrène }\end{array}$ & $x$ & $x$ \\
\hline 18 & Béton armé & $x$ & & 36 & Géotextile & $x$ & $x$ \\
\hline
\end{tabular}

\subsection{Protocole expérimental}

La Figure 1 schématise l'ensemble des étapes de traitement des échantillons et de lixiviation pour les différents cas.

\section{Prétraitement des échantillons}

Les échantillons ont été pré-conditionnés afin d'éliminer la contamination superficielle se trouvant sur eux. Pour cela, chaque échantillon a été nettoyé à l'eau du robinet, à l'eau osmosée et enfin rincé avec du méthanol. Par la suite, les matériaux en PVC, les membranes d'étanchéité SBS et les matériaux de drainage ont été finement 
coupés afin d'obtenir des morceaux de taille $<4 \mathrm{~mm}$. Les matériaux en béton ont été broyés à l'aide d'un mortier en acier, tamisés avec un tamis métallique de taille de maille de $2 \mathrm{~mm}$ et lyophilisés pendant $48 \mathrm{~h}$. Les pneumatiques (bande de roulement) ont été coupés en copeaux très fins. Enfin, les carrosseries et le panneau en acier ont été coupés en morceaux de $3,5 \times 3,5 \mathrm{~cm}$ (pour les essais de lixiviation au méthanol) et de $5 \times 3 \mathrm{~cm}$ (pour les essais de lixiviation à l'eau). Tous les échantillons ont été ensuite stockés dans des bouteilles en verre brun jusqu'à leur utilisation. Les échantillons lyophilisés sont conservés en chambre froide à $4{ }^{\circ} \mathrm{C}$.

\section{Protocole de lixiviation}

Les essais de lixiviation au méthanol et à l'eau ont été réalisés en conditions statiques. À l'exception des matériaux de drainage, des carrosseries et de la plaque en acier, le ratio liquide solide (L/S) employé a été de 10. Ce ratio est préconisé par la norme NF EN 12457-2 (AFNOR 2002) et employé par d'autres études de lixiviation en conditions statiques effectuées sur des matériaux de construction (Legret et al. 2005; Vahčič et al. 2008). Les essais de lixiviation au méthanol ont été conduits sur 10 grammes d'échantillon mis en contact avec $100 \mathrm{~mL}$ de méthanol. Pour les essais de lixiviation à l'eau, la masse de matériau et le volume d'eau utilisés ont été multipliés par cinq afin d'avoir un volume d'eau suffisant pour l'extraction des AP et BPA, et d'effectuer des analyses complémentaires ( $\mathrm{pH}$, conductivité et carbone organique dissous).

Le ratio L/S a dû être modifié lors des essais conduits sur les matériaux ayant une masse volumique faible (couche de drainage en polystyrène, natte de drainage PO/PE et géotextile). Ainsi, le ratio L/S employé lors des essais de lixiviation au méthanol a été de 80 pour la couche de drainage en polystyrène $(1 \mathrm{~g}$ de polystyrène dans $80 \mathrm{~mL}$ du méthanol) et de 20 pour la natte de drainage PO/PE et le géotextile ( $5 \mathrm{~g}$ de natte de drainage dans $100 \mathrm{~mL}$ de méthanol et $1 \mathrm{~g}$ de géotextile dans $20 \mathrm{~mL}$ de méthanol). Pour les essais de lixiviation à l'eau le ratio L/S utilisé a été de 60 pour la couche de drainage en polystyrène $(9 \mathrm{~g}$ de polystyrène en $527 \mathrm{~mL}$ d'eau) et de 50 pour le géotextile (20 g de matériaux en $1000 \mathrm{~mL}$ d'eau). Pour les carrosseries et la plaque en acier, nous avons employé un ratio liquide/surface (L/Surf). Les ratios L/Surf utilisés pour les essais de lixiviation au méthanol et à l'eau ont été respectivement de $1,6 \mathrm{~mL} / \mathrm{cm}^{2}\left(12 \mathrm{~cm}^{2}\right.$ d'échantillon en $20 \mathrm{~mL}$ du méthanol) et $8 \mathrm{~mL} / \mathrm{cm}^{2}\left(60 \mathrm{~cm}^{2}\right.$ de matériaux en contact avec $500 \mathrm{~mL}$ d'eau). Tous les essais se sont déroulés en flacons bruns, à température ambiante $\left(20^{\circ} \mathrm{C}\right)$ et sous agitation $(24 \mathrm{~h}$ pour la lixiviation au méthanol et $48 \mathrm{~h}$ pour la lixiviation à l'eau). Un blanc (eau ultrapure mise dans les mêmes conditions expérimentales) a été réalisé lors de chaque série d'essais. 


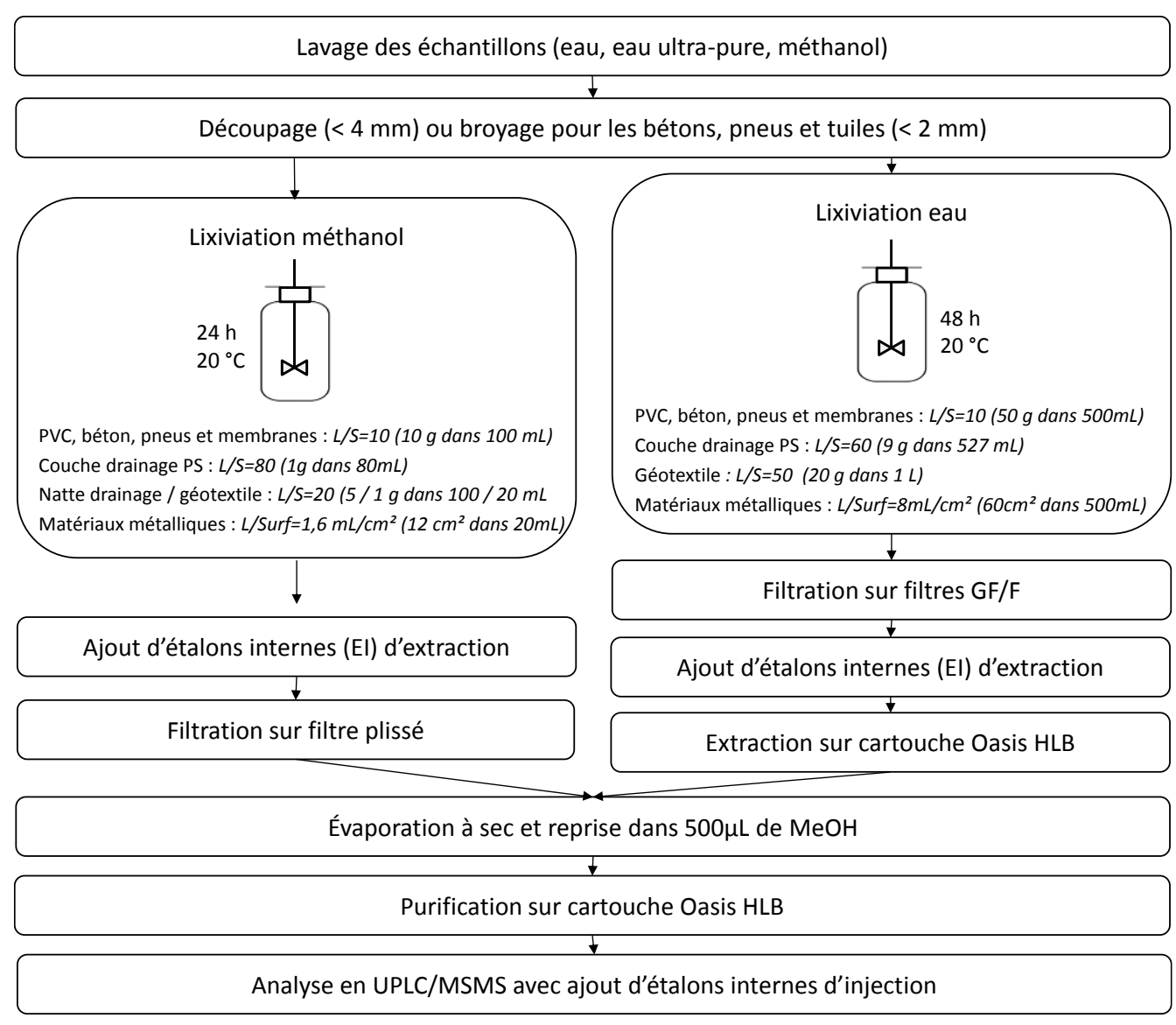

Figure 1 : Protocole de lixiviation et de traitement des échantillons

\section{Traitement de l'échantillon, extraction et analyse des AP et BPA}

Lixiviation au méthanol : les solutions de méthanol sont dopées par ajout de $50 \mu \mathrm{L}$ d'étalon interne d'extraction composé de 3 molécules deutérées (BPA-d6, NP1EO-d2 et n-OP-d17). La totalité du lixiviat est ensuite filtrée sur un filtre préalablement lavé au dichlorométhane par sonication pendant 10 minutes puis évaporé à l'évaporateur rotatif jusqu'à un volume final d'environ $2 \mathrm{~mL}$ et ensuite évaporé à sec sous flux d'azote. L'échantillon est repris dans $500 \mu \mathrm{L}$ d'un mélange eau/méthanol (50/50, v/v) pour être ensuite purifié. La purification est effectuée en phase solide (SPE) sur des cartouches Oasis ${ }^{\circledR} \mathrm{HLB}(200 \mathrm{mg} / 6 \mathrm{~mL})$. Le conditionnement des cartouches est réalisé avec $3 \mathrm{~mL}$ de méthanol et $3 \mathrm{~mL}$ d’un mélange eau/méthanol $(80 / 20 \mathrm{v} / \mathrm{v})$. Les échantillons sont ensuite chargés sur la cartouche. Après un lavage avec $3 \mathrm{~mL}$ d'eau, les cartouches sont séchées pendant 30 minutes sous flux d'azote puis éluées avec $9 \mathrm{~mL}$ d'un mélange méthanol / dichlorométhane / acétate d'éthyle (40/40/20, v/v/v). Les extraits sont conservés au congélateur jusqu'à analyse.

Lixiviation à l'eau : Les lixiviats ont été filtrés sous vide sur une membrane filtrante GF/F en fibres de verre de diamètre de $90 \mathrm{~mm}$ et de porosité nominale de 0,7 $\mu \mathrm{m}$. À partir de l'échantillon filtré nous avons effectué des mesures de $\mathrm{pH}$, conductivité, carbone organique dissous (COD), alkylphénols et bisphénol A. L'extraction des AP et BPA est réalisée en suivant le protocole mis en place par Cladière et al. (2013) par SPE après ajout des étalons internes.

Analyses des AP et du BPA: Les analyses ont été effectuées par chromatographie liquide couplée à un spectromètre de masse en tandem (LC-MS/MS) en suivant le protocole décrit par Cladière et al. (2013). Les limites de quantification (LQ) et de détection (LD) ont été déterminées pour chaque molécule selon la norme NF XPT 90-210. Les valeurs de LQ utilisées pour cette étude sont : $13 \mathrm{ng} / \mathrm{L}$ pour le BPA, $14 \mathrm{ng} / \mathrm{L}$ pour le NP, $16 \mathrm{ng} / \mathrm{L}$ pour le NP1EO, $4 \mathrm{ng} / \mathrm{L}$ pour le NP2EO, $5 \mathrm{ng} / \mathrm{L}$ pour le NP1EC, $10 \mathrm{ng} / \mathrm{L}$ pour l'OP, $8 \mathrm{ng} / \mathrm{L}$ pour l'OP1EO et $8 \mathrm{ng} / \mathrm{L}$ pour l'OP2EO. 


\section{RESULTATS}

\subsection{Lixiviation au méthanol}

\section{Concentration des lixiviats}

Les résultats de lixiviation au méthanol sont rassemblés dans le Tableau 3.

Matériaux en PVC : Le BPA est le composé majoritairement quantifié dans ces matériaux suivi du NP et des NPEO, tandis que I'OP, les OPEO et le NP1EC se trouvent principalement à l'état de traces avec des concentrations proches de celles mesurées dans le blanc. La différence de concentrations entre les échantillons d'un même matériau ne semble pas être influencée par l'âge du matériau ni par le type d'application. Elle semble être liée à la formulation du matériau et au traitement qu'a pu subir celui-ci lors de son utilisation (exemple : couche de peinture à la surface extérieure de la gouttière). Ainsi, les concentrations en BPA relarguées par le volet usagé et par la gouttière peinte sont respectivement de 5 à 9 et de 4 à 7 fois supérieures à celles mesurées dans les autres échantillons en PVC testés. La présence de BPA dans les matériaux en PVC s'explique par son usage en tant qu'antioxydant dans le traitement du PVC, constituant d'un pack d'additifs pour le traitement du PVC, agent antioxydant dans la production de plastifiants utilisés dans la transformation du PVC et agent inhibiteur pendant la phase de polymérisation du PVC (avant 2003) (ECB 2010). En ce qui concerne les NPEO, la littérature rapporte leur utilisation en tant qu'additifs dispersants lors de la fabrication du PVC polymérisé en émulsion. Enfin, I'usage de NP comme stabilisant des matières plastiques a été rapporté (ECB 2002).

Matériaux en béton : Les NPEO et le NP sont de loin les composés émis majoritairement. Les concentrations en AP dans les lixiviats des différents types de béton sont très variables entre les échantillons (de 110 à $2300 \mathrm{ng} / \mathrm{L}$ pour le NP, de 53 à 10000 ng/L pour le NP1EO et de 17 à 9200 ng/L pour le NP2EO). Les bétons de trottoir sont les bétons avec les plus fortes concentrations en NP et NPEO. La présence de NPEO dans les échantillons de béton peut être attribuée à l'utilisation des NPEO lors de la fabrication du béton en tant qu'adjuvant " entraîneur d'air » (ECB 2002; Togerö 2005; Månsson et al. 2008; INERIS 2011). En ce qui concerne le NP et le NP1EC, leur présence peut donc être attribuée d'une part à la dégradation des NPEO en condition aérobie (Maguire 1999) et d'autre part aux apports de l'environnement extérieur (absorption des composés se trouvant dans l'air et/ou la pluie). Pour les autres composés, aucune information n'a été publiée sur leur usage dans le domaine de la production des bétons.

Matériaux de drainage : Le NP reste de loin le composé lessivé aux plus fortes concentrations ( $530 \mathrm{ng} / \mathrm{L}$ pour la couche de drainage en polystyrène, $1400 \mathrm{ng} / \mathrm{L}$ pour le géotextile, $1300 \mathrm{ng} / \mathrm{L}$ pour la couche de drainage en polypropylène et $12000 \mathrm{ng} / \mathrm{L}$ pour la natte de drainage PO/PE). Ces résultats ne sont pas surprenants. En effet, les principales applications du NP et de ses dérivés sont liées à l'industrie des plastiques. Ainsi, le NP est employé en tant que stabilisant de matières plastiques et dans la fabrication des NPEO qui sont à leur tour employés comme additifs dispersants lors de la fabrication des matières plastiques polymérisées par émulsion. II est également utilisé dans la production du TNPP lequel sert comme antioxydant secondaire dans les formulations de polymères (polyéthylène, polystyrène, PVC...) et comme stabilisateur pour certains polymères (vinyliques, polyoléfine, polystyrène, polycarbonate). Nous pouvons toutefois supposer que la contribution en NP par le TNPP et les NPEO est nettement inférieure à celle apportée par son usage direct en tant que stabilisant (INERIS 2011).

L'OP est également détecté dans ces matériaux ( $28 \mathrm{ng} / \mathrm{L}$ pour la couche de drainage en polystyrène, $84 \mathrm{ng} / \mathrm{L}$ pour le géotextile, $44 \mathrm{ng} / \mathrm{L}$ pour la couche de drainage en polypropylène et $570 \mathrm{ng} / \mathrm{L}$ pour la natte de drainage $\mathrm{PO} / \mathrm{PE}$ ). $\mathrm{Au}$ regard des concentrations mesurées en NP, la proportion d'OP relarguée est de l'ordre de 2 à $5 \%$. La littérature rapporte la production non volontaire d'OP lors de la synthèse du NP commercial à une hauteur de 3 à $5 \%$ (OSPAR Commission 2003). Les NPEO sont émis par la couche de drainage en polystyrène (NP1EO : $120 \mathrm{ng} / \mathrm{L}, \mathrm{NP2EO}: 110 \mathrm{ng} / \mathrm{L}$ ), le géotextile (NP1EO : $420 \mathrm{ng} / \mathrm{L}$, NP2EO : $86 \mathrm{ng} / \mathrm{L}$ ) et dans la natte de drainage en PO/PE (NP1EO : $910 \mathrm{ng} / \mathrm{L}, \mathrm{NP} 2 \mathrm{EO}: 42 \mathrm{ng} / \mathrm{L}$ ) tandis que, les OPEO sont émis uniquement par la natte de drainage en PO/PE (OP1EO : $190 \mathrm{ng} / \mathrm{L}$, OP2EO : $72 \mathrm{ng} / \mathrm{L}$ ). La littérature rapporte l'utilisation des OPEO en tant qu'agents émulsifiants pour la synthèse de polymères et des NPEO comme additifs dispersants lors de la fabrication des matières plastiques polymérisées par émulsion (INERIS 2011; INERIS 2016). 
Le BPA a été quantifié uniquement pour 3 des 4 matériaux analysés (drainage en polyproplylène, natte PO/PE et géotextile). Les concentrations en BPA sont respectivement de 650, 1300 et $220 \mathrm{ng} / \mathrm{L}$. Plusieurs rapports scientifiques signalent l'utilisation du BPA en tant que monomère dans la synthèse de polymères (polyester carbonate, polyarylates, polysulfones, polyols, entre autres) (ECSC 2008; INERIS 2010; ANSES 2011; Nelson and Long 2012). Les matériaux de drainage sont constitués majoritairement par des produits synthétiques polymériques. Nous supposons donc que les polymères constitutifs du géotextile, la couche de drainage et la natte de drainage sont à l'origine du relargage du BPA.

Membranes d'étanchéité SBS : Le BPA, le NP, les NPEO et l'OP ont été émis par les 3 membranes testées. Le NP est le composé mesuré aux plus fortes concentrations, avec des valeurs qui fluctuent entre 40 et $230 \mu \mathrm{g} / \mathrm{L}$. Pour le BPA, le NP1EO, le NP2EO et l'OP les valeurs de concentration varient respectivement entre 1,3 et $2,3 \mu \mathrm{g} / \mathrm{L}$; 0,08 et $0,5 \mu \mathrm{g} / \mathrm{L} ; 0,3$ et $1,1 \mu \mathrm{g} / \mathrm{L}$ et 0,3 et $1,3 \mu \mathrm{g} / \mathrm{L}$. Pour les OPEO et le NP1EC les concentrations ont été généralement inférieures aux limites de quantification. La présence de NP dans ces matériaux peut s'expliquer par leur utilisation lors de la fabrication de sulfates d'éther de nonylphénol. Ce dernier intervient comme émulsifiant dans la fabrication du monomère styrène et styrène butadiène (SBR) (ECB 2002). Pour le BPA, I'OP et le NPEO, la littérature consultée ne rapporte pas d'information sur leur usage dans ce domaine.

Pneumatiques: Le NP, les NPEO, l'OP et le BPA ont été détectés dans les lixiviats des pneumatiques. Les concentrations en OPEO and NP1EC ont été généralement inférieures aux limites de quantification. L'OP est le composé majoritaire (290 et $28000 \mathrm{ng} / \mathrm{L}$ ) suivi du NP (<LQ et $6500 \mathrm{ng} / \mathrm{L}$ ), du BPA (1 100 et $4400 \mathrm{ng} / \mathrm{L}$ ), du NP1EO (<LQ et $600 \mathrm{ng} / \mathrm{L}$ ), du NP2EO (87 et $350 \mathrm{ng} / \mathrm{L}$ ) et du NP1EC (<LQ et $120 \mathrm{ng} / \mathrm{L}$ ). Cependant, ces valeurs doivent être utilisées avec précaution du fait des faibles rendements d'extraction obtenus lors des analyses.

L'utilisation des AP et du BPA dans la production des pneumatiques est largement citée dans la littérature (ECB 2002; OSPAR Commission 2003; D.E.F.R.A. 2006; Edeskär 2006; ECB 2010; INERIS 2010; INERIS 2011; INERIS 2016). L'OP est employé en tant qu'intermédiaire dans la fabrication des résines phénoliques. Ces résines sont utilisées comme agents d'adhérence dans le caoutchouc des pneumatiques. Selon le rapport OSPAR (2003), elles sont ajoutées au caoutchouc à hauteur de $1,5 \%$, ce qui correspondrait à une concentration maximale d'OP dans les pneus de $0,3 \%$. L'ETRMA (2011) indique cependant qu'un pneu moyen européen ne contiendrait que entre 0,007 et $0,012 \%$ d'impuretés en OP, présent dans certaines zones internes du pneu uniquement. Le NP intervient dans la production des résines phénoliques en tant que matière première « monomère » et dans la production du TNPP qui à son tour est utilisé comme stabilisateur pour les caoutchoucs synthétiques. On peut donc supposer que le NP présent dans les pneumatiques est celui n'ayant pas réagi lors de la polymérisation de la résine, celui présent à l'état d'impureté dans le TNPP (à hauteur de $3 \%$ ) (ECB 2002) ou celui formé par hydrolyse du TNPP (ECB 2002; ECB 2010). La présence de NPEO peut être attribuée à son utilisation en tant qu'additifs dispersants lors de la fabrication des caoutchoucs polymérisés en émulsion (caoutchouc SBR)(ECB 2002). La présence du BPA, peut s'expliquer par leur utilisation comme antioxydant dans la matrice polymère des pneus (ECB 2010).

Plaque en acier laqué et carrosseries : De fortes concentrations en BPA ont été mesurées dans les lixiviats de la plaque en acier $(16 \mu \mathrm{g} / \mathrm{L})$ et surtout des échantillons de carrosserie $(240-1200 \mu \mathrm{g} / \mathrm{L})$. Ces matériaux ont également émis du NP1EO (0,1 - 0,6 $\mu \mathrm{g} / \mathrm{L})$, du NP2EO (0,1 - $17 \mu \mathrm{g} / \mathrm{L})$, du NP (<LQ - 5,0 $\mu \mathrm{g} / \mathrm{L})$, de l'OP (0,1 - 2,3 $\mu \mathrm{g} / \mathrm{L})$ et du NP1EC (<LQ - 4,9 $\mu \mathrm{g} / \mathrm{L})$. Les concentrations en OPEO ont été faibles et généralement inférieures aux limites de quantification.

La présence du BPA peut être attribuée principalement à l'usage direct du BPA dans les durcisseurs des peintures et dans la synthèse de polyols (ANSES 2011). Les polyols sont employés pour la fabrication des résines polyuréthane lesquelles sont souvent utilisées dans les bases pigmentées et les vernis appliqués dans la couche de finition des carrosseries (Laout 2009). Les NPEO sont utilisés dans les laques et vernis comme agents mouillants, émulsifiants et dispersants (ECB 2002; INERIS 2011). On peut donc supposer que ces applications sont à l'origine des NPEO dans nos échantillons. En ce qui concerne le NP et le NP1EC, leur présence peut être expliquée par la dégradation en condition aérobie des NPEO (Maguire 1999). Aucun usage direct d'OP et OPEO dans l'industrie des vernis et des laques n'est rapporté dans la littérature.

\section{Profil d'émission dans le méthanol}


La Figure 2 présente les profils d'émission des AP et du BPA pour l'ensemble des matériaux testés en lixiviation au méthanol. Ces profils d'émission ont été construits à partir des masses des composés lixiviés par gramme de matériau sec.

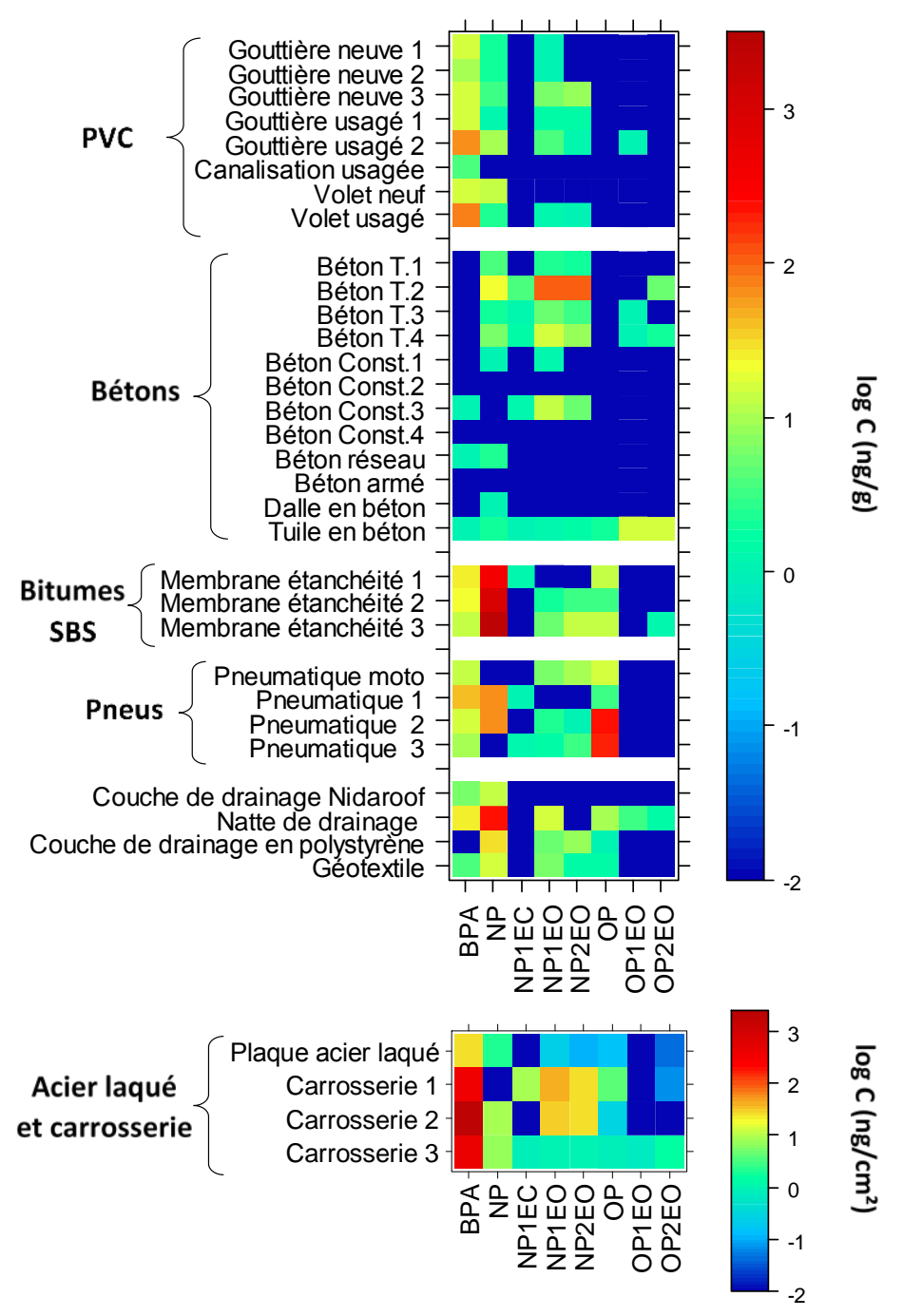

Figure 2 : Profil d'émission des AP et du BPA dans le méthanol par gramme de matériau à l'état sec ou par $\mathrm{cm}^{2}$

Le profil d'émission des AP et du BPA représenté Figure 2 montre que le BPA et le NP sont les composés avec les plus fortes émissions, tandis que les OPEO et le NP1EC présentent des émissions très faibles. Les matériaux en PVC se révèlent les plus forts émetteurs de BPA ( $9 \mathrm{ng} / \mathrm{g}$ à $70 \mathrm{ng} / \mathrm{g})$. D'autres matériaux émettent également du BPA, c'est le cas des pneumatiques (11 à $43 \mathrm{ng} / \mathrm{g}$ ), des membranes d'étanchéité SBS (14 à $23 \mathrm{ng} / \mathrm{g}$ ) et des matériaux de drainage pour toiture végétalisée (4 à $25 \mathrm{ng} / \mathrm{g}$ ). Le NP est émis majoritairement par les membranes d'étanchéité (400 à $2400 \mathrm{ng} / \mathrm{g}$ ) et en moindre mesure par les matériaux de drainage (12 à $240 \mathrm{ng} / \mathrm{g}$ ), les pneumatiques (58 à $64 \mathrm{ng} / \mathrm{g}$ ), les matériaux en PVC (1 à $12 \mathrm{ng} / \mathrm{g}$ ) et les bétons de trottoir (2 à $21 \mathrm{ng} / \mathrm{g}$ ). Les NPEO sont émis principalement par les bétons de trottoir (2 à $93 \mathrm{ng} / \mathrm{g}$ ). L'OP est relargué majoritairement par les pneumatiques (15 à $275 \mathrm{ng} / \mathrm{g}$ ). Cependant les membranes d'étanchéité (3 à $13 \mathrm{ng} / \mathrm{g}$ ) et la natte de drainage $\mathrm{PO} / \mathrm{PE}(11 \mathrm{ng} / \mathrm{g})$ se révèlent également sources d'émission d'OP.

Pour la plaque en acier et les carrosseries, dont les émissions sont imputables au revêtement de surface (laque/peinture), les émissions ont été calculées par unité de surface. Ces matériaux émettent principalement du BPA et des NPEO à des taux qui varient de 26 à $2000 \mathrm{ng} / \mathrm{cm}^{2}$ pour le BPA, de 0,2 à $39 \mathrm{ng} / \mathrm{cm}^{2}$ pour le NP1EO et de 0,1 à $27 \mathrm{ng} / \mathrm{cm}^{2}$ pour le NP2EO. 


\subsection{Lixiviation à l'eau.}

\section{Concentration des lixiviats}

Les essais de lixiviation à l'eau en conditions statiques ont été réalisés sur 21 matériaux (Tableau 2). Pour 20 des 21 matériaux testés, la présence des AP et/ou du BPA a été confirmée préalablement par les tests menés en lixiviation au méthanol. Le matériau restant est un panneau sandwich en polycarbonate. Ce matériau a été choisi en se basant sur des études scientifiques qui ont mis en évidence le relargage du BPA par les matériaux fabriqués à base de polycarbonate (Yamamoto and Yasuhara 1999; Loyo-Rosales et al. 2004; Le et al. 2008; Kubwabo et al. 2009; Cooper et al. 2011).

Le Tableau 4 récapitule l'ensemble de données obtenues lors des essais de lixiviation à l'eau pour les 21 matériaux testés. Les valeurs de $\mathrm{pH}$ des lixiviats des matériaux de drainage, des carrosseries, de la plaque en acier, de la plaque en polycarbonate et des pneumatiques sont neutres et similaires aux valeurs de $\mathrm{pH}$ des blancs (entre 5,4 et 6,8 avec une moyenne de 6,2). Par contre, les gouttières en PVC, les volets en PVC, les bétons de trottoir et les membranes d'étanchéité induisent dans l'eau un $\mathrm{pH}$ basique. Les plus fortes valeurs de $\mathrm{pH}$ sont mesurées dans les échantillons issus de la lixiviation des bétons de trottoir $(\mathrm{pH}=12)$. La mesure de la conductivité apporte une information globale sur la charge minérale relarguée par les différents matériaux soumis aux tests de lixiviation. La conductivité des blancs (comprise entre 2,0 et $16 \mu \mathrm{S} / \mathrm{cm}$ ) est modifiée par la plupart des matériaux, les bétons étant les matériaux qui génèrent les plus fortes concentrations en ions (de 4800 à $5800 \mu \mathrm{S} / \mathrm{cm}$ ), suivis des membranes SBS et des pneumatiques (respectivement de 81 à $130 \mu \mathrm{S} / \mathrm{cm}$ et de 60 à $78 \mu \mathrm{S} / \mathrm{cm}$ ). Les échantillons issus de la lixiviation des matériaux en PVC présentent un relargage moindre d'espèces chargées avec des valeurs de conductivité se situant entre 32 et $64 \mu \mathrm{S} / \mathrm{cm}$. Pour la plaque en polycarbonate, la plaque en acier, les carrosseries et les matériaux de drainage, les valeurs de conductivité restent similaires aux valeurs du blanc.

La matière organique relarguée par les différents matériaux testés a été évaluée à partir de la mesure du COD. Les résultats obtenus montrent que la plupart des matériaux testés relargue de la matière organique avec des concentrations de COD dans les lixiviats qui dépassent les valeurs des blancs (entre $<\mathrm{LQ}$ et $0,9 \mathrm{mgC} / \mathrm{L}$ avec une moyenne de $0,4 \mathrm{mgC} / \mathrm{L}$ ). Les pneumatiques sont de loin les matériaux qui génèrent les plus fortes concentrations en matière organique ( 36 et $46 \mathrm{mgC} / \mathrm{L}$ ). Les échantillons issus de la lixiviation des matériaux en PVC, béton et membranes d'étanchéité SBS présentent une concentration moindre de matière organique dissoute (1 à $30 \mathrm{mgC} / \mathrm{L}$ pour les $\mathrm{PVC}$, de 5,5 à $10 \mathrm{mgC} / \mathrm{L}$ pour le béton et les membranes SBS). Le relargage de matière organique dissoute pour la plaque en polycarbonate, la plaque en acier, les carrosseries et les matériaux de drainage est faible (inférieur à $1 \mathrm{mgC} / \mathrm{L}$ ).

De fortes concentrations en BPA sont relarguées par la plaque en polycarbonate (14 $500 \mathrm{ng} / \mathrm{L})$, les carrosseries (1 500 à 43000 ng/L), les pneumatiques (2 000 à 4100 ng/L) et les matériaux en PVC (390 à 2200 ng/L). Pour le $\mathrm{NP}$, des concentrations élevées sont mesurées dans les lixiviats des matériaux en PVC (150 à $660 \mathrm{ng} / \mathrm{L}$ ), les pneumatiques (200 à $790 \mathrm{ng} / \mathrm{L}$ ), les membranes d'étanchéité SBS (690 à $800 \mathrm{ng} / \mathrm{L}$ ) et les bétons de trottoir (390 à $680 \mathrm{ng} / \mathrm{L}$ ). Tandis que pour la plaque en polycarbonate, la plaque en acier, la carrosserie 3 et la couche de drainage en polystyrène, les concentrations en NP sont faibles voire similaires aux valeurs des blancs (entre 23 et $67 \mathrm{ng} / \mathrm{L}$ avec une moyenne de $30 \mathrm{ng} / \mathrm{L}$ ). Des concentrations élevées en OP sont observées dans les lixiviats des pneumatiques (170 et $900 \mathrm{ng} / \mathrm{L}$ ) et de la gouttière neuve $3(68 \mathrm{ng} / \mathrm{L})$. Les lixiviats des carrosseries et de béton de trottoir présentent des concentrations en OP de 14 à $16 \mathrm{ng} / \mathrm{L}$ et de 14 à $26 \mathrm{ng} / \mathrm{L}$. La plaque en polycarbonate, la plaque en acier et les matériaux de drainage ne relarguent pas d'OP, les concentrations restent proches des valeurs des blanc (3 à $5 \mathrm{ng} / \mathrm{L}$ ). Pour ce qui concerne les alkylphénols éthoxylés, les résultats montrent que le NPEO sont relargués par la gouttière neuve 3 (NP1EO : $500 \mathrm{ng} / \mathrm{L}$ et NP2EO : $390 \mathrm{ng} / \mathrm{L}$ ), la carrosserie 3 (NP1EO : $180 \mathrm{ng} / \mathrm{L}$ et NP2EO : $200 \mathrm{ng} / \mathrm{L}$ ), le béton de trottoir 2 (NP1EO : $99 \mathrm{ng} / \mathrm{L}$ et NP2EO : $180 \mathrm{ng} / \mathrm{L}$ ) et la plaque en polycarbonate (NP1EO : $110 \mathrm{ng} / \mathrm{L}$ ). Les OPEO sont relargués par les carrosseries 1 et 3 (OP1EO : 40 et $8 \mathrm{ng} / \mathrm{L}$, OP2EO : 58 et $17 \mathrm{ng} / \mathrm{L}$ ) et la gouttière neuve 3 (OP1EO : $12 \mathrm{ng} / \mathrm{L}$ et OP2EO : $14 \mathrm{ng} / \mathrm{L}$ ). Un fort relargage de NP1EC est observé provenant des bétons de trottoir $(210$ et $2300 \mathrm{ng} / \mathrm{L}$ ), des membranes d'étanchéité SBS (27 et $190 \mathrm{ng} / \mathrm{L})$, de la gouttière en PVC $3(180 \mathrm{ng} / \mathrm{L})$ et de la carrosserie 3 (110 ng/L). Pour ces matériaux, les 
concentrations du NP1EC sont de 2 à 3 ordres de grandeurs plus importantes que la valeur moyenne de concentration des blancs ( $3 \mathrm{ng} / \mathrm{L})$.

Les lixiviations à l'eau génèrent des concentrations en AP et BPA majoritairement inférieures à celles mesurées lors des essais de lixiviation au méthanol (seulement $11 \%$ des expérimentations ont montré une concentration supérieure dans le lixiviat à l'eau que dans le lixiviat au méthanol). De plus, lorsque les substances sont classées par rapport à leurs niveaux d'émission, le classement à partir de deux types de tests est différent. Cette différence était attendue étant donné que l'affinité des substances pour le méthanol et l'eau n'est pas la même, en lien avec leur hydrophobicité et leur polarité. Les rapports de concentrations dans les lixiviats issus des expérimentations avec le méthanol et avec l'eau ont été calculés et mis en relation avec le coefficient de partage octanol/eau des substances (Kow). Dans la majorité des cas, le rapport est supérieur à 1 montrant que l'extraction est plus efficace dans le méthanol. Ce rapport est le plus faible pour le bisphénol A qui a le plus faible log Kow et est plus élevé pour les autres substances dont le log Kow est au-dessus de 4. Aucun lien entre ces rapports et le type de matériau testé n'a été mis en évidence. Les concentrations émises dans l'eau sont cependant élevées pour plusieurs échantillons, prouvant la présence de composés extractibles dans l'eau et donc le risque de transfert lors du ruissellement. 


\begin{tabular}{|c|c|c|c|c|c|c|c|c|c|}
\hline \multirow{2}{*}{ Matériau } & \multirow{2}{*}{ Échantillon } & \multicolumn{8}{|c|}{ Concentrations (ng/L) } \\
\hline & & BPA & 4-NP & NP1EO & NP2EO & NP1EC & OP & OP1EO & OP2EO \\
\hline & Limites de quantification & 13 & 14 & 16 & 4 & 5 & 10 & 8 & 8 \\
\hline & Moyenne des blancs & 27 & 150 & 55 & 15 & 5 & 17 & 19 & $<L Q$ \\
\hline \multirow{8}{*}{ PVC } & Gouttière neuve 1 & 1600 & 330 & 150 & 77 & 22 & 26 & 19 & $<\mathrm{LQ}$ \\
\hline & Gouttière neuve 2 & 970 & 340 & 160 & 96 & 17 & 37 & $<\mathrm{LQ}$ & 12 \\
\hline & Gouttière neuve 3 & 1500 & 450 & 660 & 770 & 20 & 23 & 12 & 65 \\
\hline & Gouttière usagée 1 & 1500 & 280 & 220 & 170 & 45 & 30 & 19 & 9 \\
\hline & Gouttière usagée 2 & $7000^{*}$ & 1200 & 450 & 140 & 18 & 100 & 110 & 12 \\
\hline & Canalisation usagée & 420 & 170 & $<\mathrm{LQ}$ & 38 & $<\mathrm{LQ}$ & $<\mathrm{LQ}$ & $<\mathrm{LQ}$ & $<\mathrm{LQ}$ \\
\hline & Volet PVC neuf & 1800 & 1300 & $<\mathrm{LQ}$ & $<L Q$ & $<\mathrm{LQ}$ & $<\mathrm{LQ}$ & $<\mathrm{LQ}$ & $<\mathrm{LQ}$ \\
\hline & Volet PVC usagé & 8600 & 430 & 170 & 110 & 9 & $<\mathrm{LQ}$ & 33 & 16 \\
\hline \multirow{12}{*}{ Béton } & Béton trottoir 1 & 26 & 550 & 320 & 230 & 10 & 30 & $<\mathrm{LQ}$ & 47 \\
\hline & Béton trottoir 2 & 45 & 2300 & 10000 & $9200 *$ & 400 & 93 & $<\mathrm{LQ}$ & 530 \\
\hline & Béton trottoir 3 & 29 & 350 & 600 & 320 & 140 & 19 & 34 & $<\mathrm{LQ}$ \\
\hline & Béton trottoir 4 & 49 & 770 & 1600 & 840 & 180 & 66 & 130 & 190 \\
\hline & Béton bâtiment 1 (préfabriqué) & 43 & 250 & 180 & 85 & 14 & 23 & 18 & 9 \\
\hline & Béton bâtiment 2 & 28 & 230 & 130 & 34 & 14 & 27 & 25 & 8 \\
\hline & Béton bâtiment 3 & 130 & 210 & 1200 & 490 & 140 & 50 & 38 & 15 \\
\hline & Béton bâtiment 4 (neuf) & 130 & 380 & 100 & 19 & $<\mathrm{LQ}$ & 41 & $<\mathrm{LQ}$ & $<\mathrm{LQ}$ \\
\hline & Béton armé & 21 & 250 & 66 & 33 & $<\mathrm{LQ}$ & 29 & 19 & $<L Q$ \\
\hline & Béton réseau & 21 & 110 & 53 & 17 & $<\mathrm{LQ}$ & 17 & $<\mathrm{LQ}$ & $<\mathrm{LQ}$ \\
\hline & Dale en béton & 32 & 260 & 75 & 21 & $<\mathrm{LQ}$ & 34 & $<\mathrm{LQ}$ & $<L Q$ \\
\hline & Tuile en béton & 120 & 340 & 190 & 180 & $<\mathrm{LQ}$ & 200 & 1800 & 1700 \\
\hline \multirow{3}{*}{ Bitume SBS } & Membrane d'étanchéité 1 & 2300 & 39700 & 80 & $<\mathrm{LQ}$ & 140 & 1200 & $<\mathrm{LQ}$ & 54 \\
\hline & Membrane d'étanchéité 2 & 2000 & 89300 & 190 & 310 & $<\mathrm{LQ}$ & 300 & $<\mathrm{LQ}$ & $<\mathrm{LQ}$ \\
\hline & Revêtement d'étanchéité & 1400 & 233000 & 530 & 1100 & $<\mathrm{LQ}$ & 1300 & $<\mathrm{LQ}$ & 130 \\
\hline \multirow{4}{*}{ Pneus } & Pneumatique moto & 1300 & $<\mathrm{LQ}$ & 600 & 950 & 72 & 1500 & $<\mathrm{LQ}$ & $<\mathrm{LQ}$ \\
\hline & Pneumatique voiture 1 & 4400 & 5800 & $<\mathrm{LQ}$ & 87 & 93 & 290 & $<\mathrm{LQ}$ & $<\mathrm{LQ}$ \\
\hline & Pneumatique voiture 2 & 1700 & 6500 & 260 & 110 & $<\mathrm{LQ}$ & 27700 & $<\mathrm{LQ}$ & 51 \\
\hline & Pneumatique voiture 3 & 1100 & $<\mathrm{LQ}$ & 170 & 350 & 120 & 19700 & $<\mathrm{LQ}$ & $<L Q$ \\
\hline \multirow{3}{*}{$\begin{array}{l}\text { Matériaux de } \\
\text { drainage }\end{array}$} & Couche de drainage Nidaroof & 650 & 1300 & 62 & 18 & 14 & 44 & $<\mathrm{LQ}$ & $<L Q$ \\
\hline & Couche de drainage en polystyrène & 15 & 530 & 120 & 110 & $<\mathrm{LQ}$ & 28 & $<\mathrm{LQ}$ & $<L Q$ \\
\hline & Natte de drainage & 1300 & 12000 & 910 & 42 & 31 & 570 & 190 & 72 \\
\hline
\end{tabular}

* Concentrations potentiellement sous estimées car en limite haute de notre domaine de quantification. 
Tableau 3 (suite) : Récapitulatif des résultats des essais de lixiviation au méthanol conduits en conditions statiques sur des matériaux de construction et pièces automobiles

\begin{tabular}{|c|c|c|c|c|c|c|c|c|c|}
\hline \multirow{2}{*}{ Matériau } & \multirow{2}{*}{ Échantillon } & \multicolumn{8}{|c|}{ Concentrations (ng/L) } \\
\hline & & BPA & 4-NP & NP1EO & NP2EO & NP1EC & OP & OP1EO & OP2EO \\
\hline & Limites de quantification & 65 & 35 & 25 & 10 & 40 & 65 & 40 & 25 \\
\hline & Moyennes des blancs & $<L Q$ & 640 & 100 & $<L Q$ & $<L Q$ & $<L Q$ & $<L Q$ & $<L Q$ \\
\hline \multirow{4}{*}{$\begin{array}{l}\text { Matériaux } \\
\text { métalliques }\end{array}$} & Plaque en acier & 15700 & 1400 & 150 & 72 & $<\mathrm{LQ}$ & 91 & $<\mathrm{LQ}$ & 26 \\
\hline & Portière voiture & 236000 & $<\mathrm{LQ}$ & 23700 & 16800 & 4900 & 2300 & $<\mathrm{LQ}$ & 42 \\
\hline & Toit voiture & $1230000^{*}$ & 5000 & 17600 & 15600 & $<\mathrm{LQ}$ & 170 & $<\mathrm{LQ}$ & $<\mathrm{LQ}$ \\
\hline & Capot voiture & $254000 *$ & 4400 & 600 & 570 & 520 & 540 & 440 & 950 \\
\hline Couche filtrante & Géotextile & 220 & 1400 & 420 & 86 & $<\mathrm{LQ}$ & 84 & $<\mathrm{LQ}$ & $<\mathrm{LQ}$ \\
\hline
\end{tabular}

* Concentrations potentiellement sous estimées car en limite haute de notre domaine de quantification.

Tableau 4 : Récapitulatif des résultats des essais de lixiviation à l'eau conduits en conditions statiques sur des matériaux de construction et pièces automobiles. Valeurs de pH, conductivité $(\sigma, \mu \mathrm{S} / \mathrm{cm})$, carbone organique dissous (COD, $\mathrm{mgC} / \mathrm{L}$ ) et concentrations en AP et BPA (ng/L)

\begin{tabular}{|c|c|c|c|c|c|c|c|c|c|c|c|c|}
\hline Matériau & Échantillon & $\mathrm{pH}$ & $\sigma$ & COD & BPA & 4-NP & NP1EO & NP2EO & NP1EC & OP & OP1EO & OP2EO \\
\hline & Moyenne blancs & 6,3 & 6,0 & 0,4 & 58 & 30 & 41 & 40 & 3 & 5 & $<\mathrm{LQ}$ & 2 \\
\hline \multirow{7}{*}{ PVC } & Gouttière neuve 1 & 8,3 & 41 & 30 & 570 & 420 & 78 & 85 & 11 & 17 & $<\mathrm{LQ}$ & 5 \\
\hline & Gouttière neuve 2 & 8,3 & 59 & 8,2 & 430 & 220 & 19 & 58 & 9 & 12 & $<\mathrm{LQ}$ & 1 \\
\hline & Gouttière neuve 3 & 7,6 & 29 & 3,0 & 1000 & 660 & 500 & 390 & 180 & 68 & 12 & 14 \\
\hline & Gouttière usagée 1 & 8,4 & 32 & 20 & 1000 & 220 & 30 & 47 & 16 & 6 & $<\mathrm{LQ}$ & 2 \\
\hline & Gouttière usagée 2 & 8,1 & 64 & 8,0 & 2200 & 310 & 20 & 69 & 21 & 11 & $<\mathrm{LQ}$ & 2 \\
\hline & Volet usagé & 8,1 & 31 & 1,6 & 1500 & 250 & 23 & 35 & 16 & 7 & $<\mathrm{LQ}$ & 2 \\
\hline & Volet neuf & 8,5 & 40 & 1,1 & 390 & 150 & 28 & 43 & 15 & 10 & 6 & 3 \\
\hline \multirow{2}{*}{ Béton } & Béton trottoir 2 & 12 & 4800 & 5,5 & 820 & 680 & 99 & 180 & 2300 & 14 & $<\mathrm{LQ}$ & 7 \\
\hline & Béton trottoir 4 & 12 & 5800 & 6,1 & 102 & 390 & 52 & 57 & 210 & 26 & 9 & 7 \\
\hline \multirow{2}{*}{ Pneu } & Voiture 1 & 7,1 & 78 & 46 & 4100 & 790 & 92 & 38 & 21 & 170 & $<\mathrm{LQ}$ & $<\mathrm{LQ}$ \\
\hline & Voiture 2 & 6,7 & 60 & 36 & 2000 & 200 & $<L Q$ & 14 & $<\mathrm{LQ}$ & 900 & $<\mathrm{LQ}$ & $<\mathrm{LQ}$ \\
\hline \multirow{2}{*}{ Bitume SBS } & Membrane d'étanchéité 1 & 7,9 & 130 & 6,8 & 300 & 690 & 11 & 12 & 190 & 28 & $<\mathrm{LQ}$ & 3 \\
\hline & Membrane d'étanchéité 3 & 7,9 & 81 & 10 & 180 & 800 & 24 & 22 & 27 & 5 & $<\mathrm{LQ}$ & 1 \\
\hline Polycarbonate & Plaque en polycarbonate & 6,6 & 16 & 0,5 & 15000 & 61 & 110 & 63 & 4 & 4 & $<\mathrm{LQ}$ & 2 \\
\hline \multirow{4}{*}{$\begin{array}{l}\text { Matériaux } \\
\text { métalliques }\end{array}$} & Plaque en acier & 6,7 & 19 & 1,1 & 95 & 32 & 30 & 24 & 3 & 6 & $<\mathrm{LQ}$ & 1 \\
\hline & Portière voiture & 6,9 & 13 & 0,7 & 4000 & 110 & 33 & 60 & 17 & 16 & 40 & 58 \\
\hline & Toit voiture & 6,8 & 12 & 0,6 & 43000 & 100 & 68 & 47 & 7 & 16 & $<\mathrm{LQ}$ & 2 \\
\hline & Capot voiture & 7,0 & 7,0 & 0,9 & 1500 & 37 & 180 & 200 & 110 & 14 & 8 & 17 \\
\hline \multirow{3}{*}{$\begin{array}{l}\text { Matériaux } \\
\text { de drainage }\end{array}$} & Polypropylène & 7,0 & 19 & 0,8 & 250 & 170 & 29 & 34 & 3 & 9 & $<\mathrm{LQ}$ & 2 \\
\hline & Polystyrène & 7,2 & 12 & 0,4 & 80 & 46 & 24 & 44 & 7 & 5 & $<\mathrm{LQ}$ & 2 \\
\hline & Géotextile & 7,1 & 15 & 0,3 & 230 & 88 & 23 & 37 & 9 & 8 & $<\mathrm{LQ}$ & 2 \\
\hline
\end{tabular}




\section{Profil d'émission dans l'eau}

La Figure 3 présente les profils d'émission des AP et du BPA pour les matériaux testés en lixiviation à l'eau. Ces profils ont été réalisés en prenant en compte les masses des composés par gramme de matériau à l'état sec ou par $\mathrm{cm}^{2}$ de matériau (pour les carrosseries et la plaque en acier).

Ces profils d'émission dans l'eau diffèrent sensiblement de ceux obtenus dans le méthanol: émissions généralement plus faibles dans l'eau que dans le méthanol, mais aussi variations dans l'ordre d'importance des composés émis par un matériau et dans l'ordre d'importance des différents matériaux vis-à-vis de l'émission d’un composé.

Le profil d'émission montre que la plaque en polycarbonate est le matériau avec la plus forte émission de BPA dans l'eau (140 ng/g). Les matériaux en PVC sont émetteurs de BPA (3,0 à $21 \mathrm{ng} / \mathrm{g}$ ) et de NP (1,0 à 6,0 ng/g). Les pneumatiques sont de loin les principaux émetteurs d'OP dans l'eau (2,0 à 9,0 ng/g). Ils émettent également du BPA (20 à $39 \mathrm{ng} / \mathrm{g}$ ) et du NP (1,5 à 7,0 ng/g). Les membranes d'étanchéité SBS présentent de fortes émissions de $\mathrm{NP}(6,0$ à 7,5 ng/g). Les bétons de trottoir sont les principaux émetteurs de NP1EC ( $22 \mathrm{ng} / \mathrm{g})$. Les matériaux de drainages émettent majoritairement du BPA (1,0 à 9,0 ng/g) et du NP (1,0 à 2,0 ng/g). Les émissions des NPEO et des OPEO sont faibles (inférieures à $1 \mathrm{ng} / \mathrm{g}$ ) pour l'ensemble de matériaux.

En ce qui concerne les carrosseries et la plaque en acier, le profil d'émission met en évidence que le BPA est le composé majoritairement émis, notamment pour les carrosseries dont les émissions varient entre 10 et $360 \mathrm{ng} / \mathrm{cm}^{2}$.

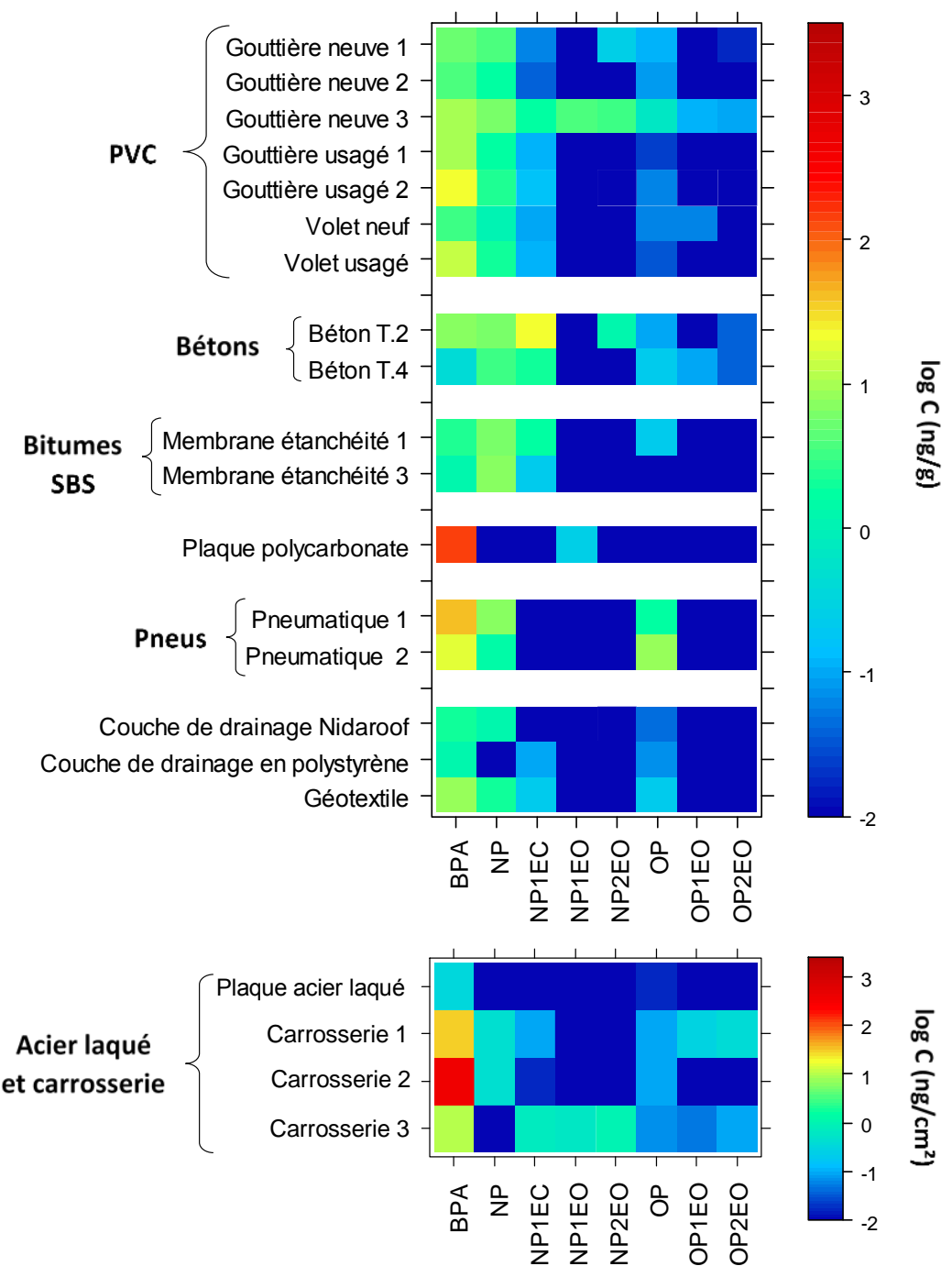

Figure 3 : Profil d'émission dans l'eau des AP et du BPA par gramme de matériau sec ou par $\mathrm{cm}^{2}$ de matériau 
Les profils d'émission dans l'eau (Figure 3), sont globalement cohérents avec les profils d'émission dans le méthanol présentés précédemment (Figure 2). Comme pour les concentrations, dans la majorité des cas, l'extraction est plus efficace dans le méthanol. La différence entre les lixiviations à l'eau et au méthanol est cependant moindre en termes de masse émise par masse de matériau qu'en termes de concentration du lixiviat (rappelons que le ratio L/S diffère entre les 2 types d'essais). Un écart important entre les émissions dans l'eau et dans le méthanol est cependant observé pour le NP et l'OP issus des membranes d'étanchéités et des pneumatiques, dont les émissions sont moins prépondérantes dans l'eau. La Figure 4 présente les rapports de masses émises dans les lixiviats issus des expérimentations avec le méthanol et avec l'eau, en fonction du log Kow. Ce rapport est le plus faible pour le bisphénol A (inférieur à 11) dont le log Kow est le plus faible, et est plus élevé pour les autres substances (entre 1 et 313) dont le log Kow dépasse 4. Les variations de Kow, de pH, de conductivité ou de COD ne sont pas corrélées avec les variations de profils d'émission. Les phénomènes mis en jeu sont donc complexes et multifactoriels, dépendant à la fois de la substance considérée, de la formulation du matériau considéré et des conditions expérimentales en ce qui concerne le solvant, le $\mathrm{pH}$, la conductivité, le COD et le rapport L/S.

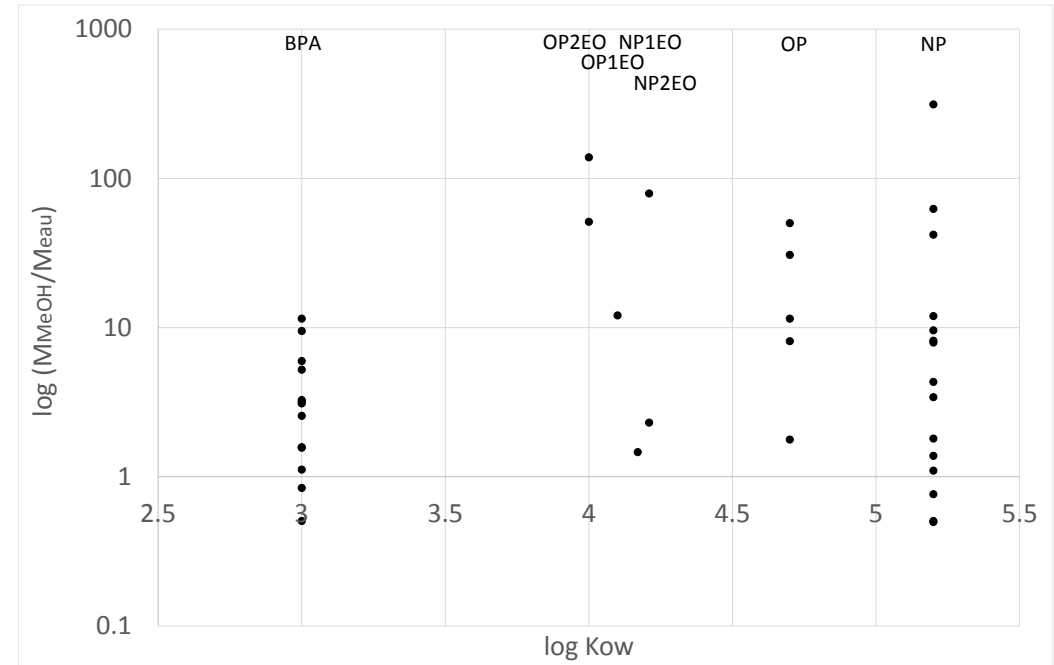

Figure 4 : Rapport (en log) des masses émises dans les lixiviats avec le méthanol et avec l'eau en fonction du log Kow des substances

\subsection{Analyse des fluides automobiles}

Les résultats de l'analyse des fluides automobiles, présentés en Tableau 5, montrent la présence de NP dans l'ensemble des fluides analysés, avec des concentrations variant de 1,1 à $7,5 \mu \mathrm{g} / \mathrm{L}$ pour les liquides de refroidissement, de 2,2 à $5,2 \mu \mathrm{g} / \mathrm{L}$ pour les lave-glaces et de 2400 à $3900 \mu \mathrm{g} / \mathrm{L}$ pour les liquides de frein. La présence de BPA est également observée dans certains échantillons. Elle est importante dans un des liquides de refroidissement $(1700 \mu \mathrm{g} / \mathrm{L})$ et extrêmement élevée dans les trois liquides de frein (0,3 à 5,5 g/L). Le BPA est en effet ajouté à des concentrations $<1 \%$ à des liquides de frein en tant qu'antioxydant (ANSES 2011) et l'analyse des fiches de données sécurité confirme souvent cette présence.

Tableau 5 : Concentrations en AP et BPA de différents fluides automobiles ( $\mu \mathrm{g} / \mathrm{L})$

\begin{tabular}{lccccccc}
\hline Échantillons & BPA & 4-NP & NP1EO & NP2EO & OP & OP1EO & OP2EO \\
\hline Blanc & 0,14 & 0,40 & 0,20 & 0,20 & $<L D$ & $<$ LD & 0,06 \\
\hline \multirow{3}{*}{ Liquides de } & 0,12 & 1,1 & $<\mathrm{LD}$ & $<\mathrm{LQ}$ & $<\mathrm{LQ}$ & $<\mathrm{LQ}$ & $<\mathrm{LD}$ \\
refroidissement & 0,45 & 1,4 & $<\mathrm{LD}$ & $<\mathrm{LQ}$ & $<\mathrm{LQ}$ & $<\mathrm{LQ}$ & $<\mathrm{LD}$ \\
& 1700 & 6,1 & 4,4 & 4,2 & 56 & $<\mathrm{LQ}$ & 8,0 \\
& 0,40 & 7,5 & $<\mathrm{LD}$ & $<\mathrm{LQ}$ & 0,54 & $<\mathrm{LQ}$ & $<\mathrm{LQ}$ \\
\hline \multirow{4}{*}{ Lave-glaces } & 0,39 & 2,2 & $<\mathrm{LD}$ & $<\mathrm{LQ}$ & $<\mathrm{LQ}$ & $<\mathrm{LQ}$ & $<\mathrm{LD}$ \\
& 0,35 & 5,1 & $<\mathrm{LD}$ & $<\mathrm{LQ}$ & 1,1 & $<\mathrm{LQ}$ & $<\mathrm{LD}$ \\
& $<\mathrm{LQ}$ & 3,5 & $<\mathrm{LD}$ & $<\mathrm{LQ}$ & $<\mathrm{LQ}$ & $<\mathrm{LQ}$ & $<\mathrm{LD}$ \\
& 2,43 & 3,2 & $<\mathrm{LD}$ & $<\mathrm{LD}$ & $<\mathrm{LQ}$ & $<\mathrm{LQ}$ & $<\mathrm{LD}$ \\
\hline \multirow{4}{*}{ Liquides de frein } & $5,5.10^{6}$ & 2500 & $<\mathrm{LD}$ & $<\mathrm{LD}$ & 4100 & $<\mathrm{LQ}$ & $<\mathrm{LD}$ \\
& $5,3.10^{6}$ & 3900 & $<\mathrm{LD}$ & 228 & $<\mathrm{LQ}$ & $<\mathrm{LQ}$ & $<\mathrm{LD}$ \\
& $2,8.10^{5}$ & 2400 & $<\mathrm{LD}$ & $<\mathrm{LQ}$ & $<\mathrm{LQ}$ & $<\mathrm{LQ}$ & $<\mathrm{LQ}$ \\
\hline
\end{tabular}




\section{CONCLUSIONS}

Dans cette étude portant sur le potentiel d'émission d'alkylphénols et de bisphénol A dans les eaux de ruissellement urbaines, les essais de lixiviation au méthanol réalisés sur 29 matériaux de construction et 7 matériaux automobiles ont permis de détecter la présence d'au moins un des composés recherchés (BPA, NP, NP1EC, NP1EO, NP2EO, OP, OP1EO, OP2EO) à des niveaux significativement supérieurs aux blancs dans la plupart des échantillons testés, démontrant la présence ubiquiste de ces composés dans les matériaux urbains. Les essais de lixiviation dans l'eau ont permis d'identifier les matériaux dans lesquels les AP et le BPA peuvent être extractibles directement dans l'eau et qui risquent donc de contaminer le ruissellement lors des événements pluvieux, et de hiérarchiser ces matériaux par rapport à leur potentiel émissif.

Des différences importantes dans les émissions sont observées entre des échantillons d'un même type de matériau. Cette variabilité reflète probablement la diversité des formulations et des procédés de fabrication, ainsi que les apports de l'environnement extérieur (cas de certains matériaux anciens et poreux comme les bétons qui ont pu accumuler des AP et/ou du BPA se trouvant dans l'air et/ou la pluie).

Les composés émis le plus fréquemment lors de la lixiviation à l'eau sont le bisphénol A et le nonylphénol. Les émissions de bisphénol A les plus importantes ont été mesurées pour le polycarbonate $(140 \mathrm{ng} / \mathrm{g})$, les pneumatiques (20 à $39 \mathrm{ng} / \mathrm{g}$ ), et certaines carrosseries de voiture (10 à $\left.300 \mathrm{ng} / \mathrm{cm}^{2}\right)$. Le nonylphénol a été lixivié en quantités importantes ( 1 à $10 \mathrm{ng} / \mathrm{g}$ ) pour les PVC, certains bétons et les membranes d'étanchéités SBS. Les pneumatiques sont les seuls matériaux présentant de fortes émissions en octylphénol (1 à $10 \mathrm{ng} / \mathrm{g}$ ). Cependant, la formulation des pneumatiques évolue. Il s'avère donc indispensable de mener d'autres études comme cellesci sur des pneumatiques neufs afin de vérifier l'émission d'octylphénol dans les nouvelles formulations. Parmi les trois types de fluides automobiles étudiés (liquide de frein, lave-glace, liquide de refroidissement), les fuites de liquide de frein pourraient s'avérer une source de bisphénol A et dans une moindre mesure de nonylphénol.

Les résultats de ce travail permettent d'identifier les principales sources primaires pouvant expliquer la contamination des eaux de ruissellement en alkylphénols et en bisphénol $A$, et de les hiérarchiser. Les conditions des essais en lixiviation sont cependant limitantes et très différentes des conditions de ruissellement lors d'une pluie (surface de contact et temps de contact lors d'une pluie beaucoup plus faibles que dans le cas de la lixiviation) et ne permettent pas de préjuger des concentrations pouvant être émises dans le ruissellement. D’autre part, les essais de lixiviation ont été menés sur un nombre limité d'échantillons et dans certains cas uniquement réalisés sur des matériaux neufs ou anciens. Il semblerait judicieux d'élargir le nombre d'échantillons et de tester pour chaque type de matériaux des échantillons neufs et usagés afin de mieux évaluer l'émission des AP et BPA en fonction de l'évolution des formulations. Par ailleurs, des essais sous conditions de pluies simulées sont envisagés pour mieux évaluer les facteurs d'émission dans le ruissellement.

Cette étude est une première ouverture à la recherche de sources d'émission des AP et BPA dans les zones urbaines, notamment en ce qui concerne le secteur automobile et les infrastructures urbaines. II semblerait nécessaire d'aller vers une évaluation environnementale systématique des matériaux et des produits avant la commercialisation. Cette évaluation pourrait intégrer d'une part la fabrication et la fin de la vie, et d'autre part, les émissions dans l'environnement pendant la durée totale de l'utilisation des matériaux. À ce jour les évaluations environnementales se focalisent uniquement sur la fabrication et sur la fin de vie des matériaux. 


\section{Références bibiographiques}

AFNOR (2002). Norme NF EN 12457-2 - Caractérisation des déchets - Lixiviation - Essai de conformité pour lixiviation des déchets fragmentés et des boues - Partie 2 : essai en bâchée unique avec un rapport liquide-solide de $10 \mathrm{l} / \mathrm{kg}$ et une granularité inférieure à $4 \mathrm{~mm}$ (sans ou avec réduction de la granularité) datant de décembre 2002.

ANSES (2011). 'Health effects of bisphenol A. French Agency for Food, Environmental and Occupational Health and Safety - ANSES'. (French: In.)

Becouze-Lareure, C. (2010). Caractérisation et estimation des flux de substances prioritaires dans les rejets urbains par temps de pluie sur deux bassins versants expérimentaux. INSA de Lyon In.

Benachour, N., and Aris, A. (2009). Toxic effects of low doses of Bisphenol-A on human placental cells. Toxicology and Applied Pharmacology 241, 322-328. doi:10.1016/j.taap.2009.09.005

Björklund, K., Cousins, A. P., Strömvall, A.-M., and Malmqvist, P.-A. (2009). Phthalates and nonylphenols in urban runoff: Occurrence, distribution and area emission factors. Science of The Total Environment 407, 46654672. doi:10.1016/j.scitotenv.2009.04.040

Bressy, A., Gromaire, M. C., Lorgeoux, C., and Chebbo, G. (2011). Alkylphenols in atmospheric depositions and urban runoff. Water Science and Technology 63, 671-679. doi:10.2166/wst.2011.121

Bressy, A., Gromaire, M. C., Lorgeoux, C., Saad, M., Leroy, F., and Chebbo, G. (2012). Towards the determination of an optimal scale for stormwater quality management: Micropollutants in a small residential catchment. Water Research 46, 6799-6810. doi:10.1016/j.watres.2011.12.017

Cladière, M., Bonhomme, C., Vilmin, L., Gasperi, J., Flipo, N., Habets, F., and Tassin, B. (2013). Modelling the fate of nonylphenolic compounds in the Seine River - part 2: Assessing the impact of global change on daily concentrations. Science of the Total Environment 468-469, 1059-1068. doi:10.1016/j.scitotenv.2013.09.029

Cooper, J. E., Kendig, E. L., and Belcher, S. M. (2011). Assessment of bisphenol A released from reusable plastic, aluminium and stainless steel water bottles. Chemosphere 85, 943-947. doi:10.1016/j.chemosphere.2011.06.060

D.E.F.R.A. (2006). '4-tert-Octylphenol Risk Reduction Strategy and Analysis of Advantages and Drawbacks. Final report. DEFRA. Department for Environment, Food and Rural Affairs'.

Diehl, G., Wasinski, F. A. H., Roberz, B., Luftmann, H., Schmidt, T. C., Andersson, J. T., and Karst, U. (2004). Liquid Chromatography/Electrochemistry/Mass Spectrometry as Screening Technique for Alcohols and Phenols in Fuels. Microchimica Acta 146, 137-147. doi:10.1007/s00604-004-0202-x

ECB (2002). 'European Union Risk Assessment Report: 4-nonylphenol (branched) and nonylphenol'. (European Chemicals Bureau.)

ECB (2010). 'Updated European Union risk assessment report: 4,4'-isopropylidenediphenol (bisphenol-A) : environment addendum of February 2008.' (Publications Office: Luxembourg.) Available at: http://dx.publications.europa.eu/10.2788/40195 [accessed 11 May 2017]

ECSC (2008). Évaluation préalable finale pour le Défi concernant le Phénol, 4,4'-(1-méthyléthylidène)bis (Bisphénol-A). Environnement Canada Santé Canada.

Edeskär, T. (2006). Use of Tyre Shreds in Civil Engineering Applications. Technical and Environmental Properties. Luleå University of Technology.

Eriksson, E., Baun, A., Scholes, L., Ledin, A., Ahlman, S., Revitt, M., Noutsopoulos, C., and Mikkelsen, P. S. (2007). Selected stormwater priority pollutants - a European perspective. Science of the Total Environment 383, 41-51.

ETRMA (2011). Comments for Annex XV-SVHC dossier for the 4 (para)-ter-octylphenol.

Fries, E., and Püttmann, W. (2004). Occurrence of 4-Nonylphenol in rain and snow. Atmospheric Environment 38, 2013-2016. doi:10.1016/j.atmosenv.2004.01.013

Gasperi, J. (ce numéro). Contamination des eaux pluviales par les micropolluants : avancées du projet INOGEV. Techniques Sciences et Méthodes.

Gasperi, J., Sebastian, C., Ruban, V., Delamain, M., Percot, S., Wiest, L., Mirande, C., Caupos, E., Demare, D., Kessoo, M. D., Saad, M., Schwartz, J. J., Dubois, P., Fratta, C., Wolff, H., Moilleron, R., Chebbo, G., Cren, C., Millet, M., Barraud, S., and Gromaire, M. C. (2014). Micropollutants in urban stormwater: occurrence, 
concentrations, and atmospheric contributions for a wide range of contaminants in three French catchments. Environmental Science and Pollution Research 21, 5267-5281. doi:10.1007/s11356-0132396-0

Harréus, U. A., Wallner, B. C., Kastenbauer, E. R., and Kleinsasser, N. H. (2002). Genotoxicity and Cytotoxicity of 4-Nonylphenol Ethoxylate on Lymphocytes as Assessed by the Comet Assay. International Journal of Environmental Analytical Chemistry 82, 395-401. doi:10.1080/0306731021000015047

Hellgren, A.-C., Weissenborn, P., and Holmberg, K. (1999). Surfactants in water-borne paints. Progress in Organic Coatings 35, 79-87. doi:10.1016/S0300-9440(99)00013-2

INERIS (2010). Données technico-économiques sur les substances chimiques en France : Bisphénol A. DRC-10102861-01251B.

INERIS (2011). Données technico-économiques sur les substances chimiques en France : Nonylphénols. DRC-11118962-11079A.

INERIS (2016). Données technico-économiques sur les substances chimiques en France: Octylphénols et éthoxylates. DRC-16-158744-11472A.

Kubwabo, C., Kosarac, I., Stewart, B., Gauthier, B. R., Lalonde, K., and Lalonde, P. J. (2009). Migration of bisphenol A from plastic baby bottles, baby bottle liners and reusable polycarbonate drinking bottles. Food Additives \& Contaminants: Part A 26, 928-937. doi:10.1080/02652030802706725

Laout, J. C. (2009). Formulation des peintures - Physico-chimie et matières pulvérulentes. Techniques de l'ingénieur.

Le, H. H., Carlson, E. M., Chua, J. P., and Belcher, S. M. (2008). Bisphenol A is released from polycarbonate drinking bottles and mimics the neurotoxic actions of estrogen in developing cerebellar neurons. Toxicology Letters 176, 149-156. doi:10.1016/j.toxlet.2007.11.001

Legret, M., Odie, L., Demare, D., and Jullien, A. (2005). Leaching of heavy metals and polycyclic aromatic hydrocarbons from reclaimed asphalt pavement. Water Research 39, 3675-3685. doi:10.1016/j.watres.2005.06.017

LOI n²012-1442 (2012). 'LOI n²012-1442 du 24 décembre 2012 visant à la suspension de la fabrication, de l'importation, de l'exportation et de la mise sur le marché de tout conditionnement à vocation alimentaire contenant du bisphénol $A^{\prime}$.

Loyo-Rosales, J. E., Rosales-Rivera, G. C., Lynch, A. M., Rice, C. P., and Torrents, A. (2004). Migration of Nonylphenol from Plastic Containers to Water and a Milk Surrogate. Journal of Agricultural and Food Chemistry 52, 2016-2020. doi:10.1021/jf0345696

Maguire, R. J. (1999). Review of the persistence of nonylphénol and nonylphenol ethoxylates in aquatic environments. Water quality research journal of Canada 34, 37-78.

Månsson, N., Sörme, L., Wahlberg, C., and Bergbäck, B. (2008). Sources of Alkylphenols and Alkylphenol Ethoxylates in Wastewater-A Substance Flow Analysis in Stockholm, Sweden. Water, Air, \& Soil Pollution: Focus 8, 445-456. doi:10.1007/s11267-008-9186-6

MSI Reports Ltd (2006). Le marché des matériaux de Couverture de Toits en France. MSI. Marketing Research for Industry Ltd.

MSI Reports Ltd (2007). Le marché des vérandas en France, 2002-2011. MSI. Marketing Research for Industry Ltd.

MSI Reports Ltd (2009). Le marché des volets en France. MSI. Marketing Research for Industry Ltd.

MSI Reports Ltd (2011). Marché des fenêtres et des portes dans le secteur résidentiel en France. MSI. Marketing Research for Industry Ltd.

MSI Reports Ltd (2010). Marché des portes industrielles, commerciales et piétonnes en France. MSI. Marketing Research for Industry Ltd.

Nelson, A. M., and Long, T. E. (2012). A perspective on emerging polymer technologies for bisphenol-A replacement. Polymer International 61, 1485-1491. doi:10.1002/pi.4323

OSPAR Commission (2003). 'Octylphenol'. (OSPAR.) Available at: https://www.ospar.org/documents?v=7031 [accessed 11 May 2017]

Réglement européen 321/2011 (2011). 'Amending Regulation (EU) No 10/2011 as regards the restriction of use of Bisphenol $A$ in plastic infant feeding bottles Text with EEA relevance'. 
Rule, K. L., Comber, S. D. W., Ross, D., Thornton, A., Makropoulos, C. K., and Rautiu, R. (2006). Sources of priority substances entering an urban wastewater catchment-trace organic chemicals. Chemosphere 63, 581591. doi:10.1016/j.chemosphere.2005.08.016

Soares, A., Guieysse, B., Jefferson, B., Cartmell, E., and Lester, J. N. (2008). Nonylphenol in the environment: A critical review on occurrence, fate, toxicity and treatment in wastewaters. Environment International 34, 1033-1049. doi:10.1016/j.envint.2008.01.004

Togerö, Å. (2005). Leaching of Hazardous Substances from Additives and Admixtures in Concrete. Environmental Engineering Science 23, 102-117. doi:10.1089/ees.2006.23.102

Union européenne (2000). 'Directive 2000/60/CE du Parlement européen et du Conseil du 23 octobre 2000 établissant un cadre pour une politique communautaire dans le domaine de l'eau'.

Union européenne (2003). 'Directive Européenne n²003-53 du 18 juin 2003 du Parlement européen et du Conseil portant vingt-sixième modification de la directive 76/769/CEE du Conseil concernant la limitation de la mise sur le marché et de l'emploi de certaines substances et préparations dangereuses (nonylphénol, éthoxylate de nonylphénol et ciment)'.

Vahčič, M., Milačič, R., Mladenovič, A., Murko, S., Zuliani, T., Zupančič, M., and Ščančar, J. (2008). Leachability of $\mathrm{Cr}(\mathrm{VI})$ and other metals from asphalt composites with addition of filter dust. Pay as you throw: a tool fo urban waste management 28, 2667-2674. doi:10.1016/j.wasman.2008.01.003

Yamamoto, T., and Yasuhara, A. (1999). Quantities of bisphenol a leached from plastic waste samples. Chemosphere 38, 2569-2576. doi:10.1016/S0045-6535(98)00464-0 
TITLE

Evaluation of the potential alkylphenol et bisphenol A leaching from building materials, auto parts and consumables

\section{KEYWORDS}

Alkylphenol, Automotive materials, Bisphenol A, Building materials, Emission, Leaching, Runoff

\section{ABSTRACT}

Alkylphenols (AP) and bisphenol A (BPA) contamination of urban runoff has already been established. Potential sources of these contaminants in runoff are endogenous to the urban watershed and are related in particular to traffic and leaching from construction materials. This article summarizes the results of experimental work carried out on a selection of building materials, automotive materials and consumables which can be in contact with rain, to as to assess their potential for alkylphenols, alkylphenol ethoxylates and bisphenol A emission into the runoff. 36 samples of materials, new and used across 7 major families of building materials (PVC, concrete, polycarbonate, SBS modified bitumen, drainage materials) and automotive materials (body, tires) were subjected to leaching tests with methanol and then, for a selection of them with water. The results demonstrate the presence of AP and BPA ubiquitous in urban material and their extractable character with water. The compounds with the strongest emission rates are bisphenol $A$ and nonylphenol. The most important BPA emissions into water (10 to $300 \mathrm{ng} / \mathrm{g}$ ) were measured for polycarbonate, tires, some car bodies and PVC. Nonylphenol was leached in large quantities ( 1 to $10 \mathrm{ng} / \mathrm{g}$ ) from PVC, certain concretes, SBS modified bitumen and body samples. The tires are the only materials having a strong emission in octylphenol ( 1 to $10 \mathrm{ng} / \mathrm{g}$ ). The analysis of automotive fluids confirmed the presence of BPA ( 0.3 to $5.5 \mathrm{~g} / \mathrm{L}$ ) and nonylphenol ( 2.3 to $2.9 \mathrm{mg} / \mathrm{L}$ ) in brake fluids, while AP and BPA can be found at trace in coolants and windscreen washer. 